F. R. O'Keefe, J. A. Meachen, and P. D. Polly

2 On Rank Deficiency in Phenotypic Covariance Matrices

$4 \quad$ F. Robin O'Keefe ${ }^{1 *}$

5 Julie A. Meachen ${ }^{2}$

6 P. David Polly ${ }^{3}$

8 1. Marshall University, Biological Sciences, Huntington, WV

9 2. Des Moines University, Anatomy Department, Des Moines, IA

10 3. Indiana University, Earth and Atmospheric Sciences, Bloomington, IN

11

12 *corresponding author: F. Robin O’Keefe, Professor, Marshall University, College of

13 Science 265, One John Marshall Drive, Hunington, WV 25755. Phone: +1 3046962427.

14 Email: okeefef@marshall.edu

16 Running Head: Rank Deficiency in Phenotypic Covariance

18 Conflict of Interest Statement: The authors state that they have no conflict of interest to

19 disclose.

21 Keywords: Dire wolf, Canis dirus, geometric morphometrics, phenotypic covariance,

22 modularity and integration, information entropy, effective rank, effective dispersion,

23 relative dispersion, latent dispersion. 


\section{RANK DEFICIENCY IN PHENOTYPIC COVARIANCE}

\section{ABSTRACT}

25 This paper is concerned with rank deficiency in phenotypic covariance matrices: first to

26 establish it is a problem by measuring it, and then proposing methods to treat for it.

27 Significant rank deficiency can mislead current measures of whole-shape phenotypic

28 integration, because they rely on eigenvalues of the covariance matrix, and highly rank

29 deficient matrices will have a large percentage of meaningless eigenvalues. This paper

30 has three goals. The first is to examine a typical geometric morphometric data set and

31 establish that its covariance matrix is rank deficient. We employ the concept of

32 information, or Shannon, entropy to demonstrate that a sample of dire wolf jaws is highly

33 rank deficient. The different sources of rank deficiency are identified, and include the

34 Generalized Procrustes analysis itself, use of the correlation matrix, insufficient sample

35 size, and phenotypic covariance. Only the last of these is of biological interest.

36 Our second goal is to examine a test case where a change in integration is known,

37 allowing us to document how rank deficiency affects two measures of whole shape

38 integration (eigenvalue standard deviation and standardized generalized variance). This

39 test case utilizes the dire wolf data set from Part 1, and introduces another population that

40 is 5000 years older. Modularity models are generated and tested for both populations,

41 showing that one population is more integrated than the other. We demonstrate that

42 eigenvalue variance characterizes the integration change incorrectly, while the

43 standardized generalized variance lacks sensitivity. Both metrics are impacted by the

44 inclusion of many small eigenvalues arising from rank deficiency of the covariance

45 matrix. We propose a modification of the standardized generalized variance, again based

46 on information entropy, that considers only the eigenvalues carrying non-redundant 
F. R. O’Keefe, J. A. Meachen, and P. D. Polly

47 information. We demonstrate that this metric is successful in identifying the integration

48 change in the test case.

49 The third goal of this paper is to generalize the new metric to the case of arbitrary

50 sample size. This is done by normalizing the new metric to the amount of information

51 present in a permuted covariance matrix. We term the resulting metric the 'relative

52 dispersion', and it is sample size corrected. As a proof of concept we us the new metric to

53 compare the dire wolf data set from the first part of this paper to a third data set

54 comprising jaws of Smilodon fatalis. We demonstrate that the Smilodon jaw is much

55 more integrated than the dire wolf jaw. Finally, this information entropy-based measures

56 of integration allows comparison of whole shape integration in dense semilandmark

57 environments, allowing characterization of the information content of any given shape, a

58 quantity we term 'latent dispersion'.

59 


\section{RANK DEFICIENCY IN PHENOTYPIC COVARIANCE}

\section{0 [introduction]}

61 The study of integration in biological systems has a long history, stretching back to

62 Darwin (1859), given a modern footing by Olson and Miller (1958), quantified by Van

63 Valen (1974) and Cheverud (1982, 1996), and maturing into a broad topic of modern

64 inquiry encompassing genetics, development, and the phenotype (see Klingenberg, 2013,

65 and Goswami and Polly, 2010, for reviews). In a biological context, 'integration' means

66 that traits of a whole organism covary to a significant degree. This covariance is critical

67 in evolving populations, because traits are not free to respond to selection without

68 impacting dependent traits, and the directionality of selection response is constrained by

69 these dependencies (Grabowski and Porto, 2017, Figure 1). Yet trait dependency can also

70 remove constraint by giving a population access to novel areas of adaptive space

71 (Goswami et al., 2014, Figure 5). Consequently phenotypic integration is central to the

72 evolvability of biological systems, because it defines the achievable adaptive space.

\section{Background}

75 The use of landmark data to characterize biological shape, and to quantify shape change,

76 has become ubiquitous since the introduction of geometric morphometrics by Bookstein

77 (1997; reviewed in Zelditch et al., 2012). Analysis of geometric morphometric landmark

78 data begins with Generalized Procrustes Analysis (GPA), wherein the centroid of each

79 specimen is translated to the origin, and the coordinates are rotated and scaled to a mean

80 shape so that the intra-landmark variance among specimens is minimized (Bookstein

81 1997). Given a matrix $\mathbf{L M}_{\mathbf{n , v}}$ of $v$ two- or three-dimensional landmark coordinates and $n$

82 specimens, a matrix resulting from Procrustes superimposition is: 
F. R. O'Keefe, J. A. Meachen, and P. D. Polly

$$
x_{n, v}=G P A\left(L M_{n, v}\right)
$$

85 There are myriad examples in the literature of the application of principal

86 components analysis to GPA-transformed landmark data (for example, Segura et al.,

87 2020, for canid dentaries; Brannick et al., 2015, for dire wolf dentaries, and O'Keefe et

88 al. 2014, for dire wolf crania). The usual analysis progression is to calculate the principal

89 component scores for the first few PCs, and plot the specimens into the principal

90 component space. Principal components analysis is therefore a powerful tool for

91 dimensionality reduction, allowing the visualization and biological interpretation of a

92 large proportion of the variance on one or a few axes. Analyses of this type typically

93 utilize the first few eigenvectors, so the magnitude of their associated eigenvalues is not a

94 central concern beyond ensuring their significance. However the eigenvalue vector also

95 carries biological signal, and analysis of its distribution has given rise to the concept of

96 whole-shape phenotypic integration.

98 Whole-Shape Integration Measures

99 “The 'generalized variance', $|\Sigma|$, the determinant of the variance-co-variance matrix, $i$ s

100 related to the area (or hypervolume) of the equi- probability ellipses (ellipsoids) of the

101 distribution.”-VanValen, 1974, p. 235.

102 The distribution of the eigenvalue vector has formed the basis of several metrics intended

103 to quantify the strength of covariation in a whole shape. As stated by VanValen in the

104 quote above, the shape space containing the objects of interest can be conceptualized as a 


\section{RANK DEFICIENCY IN PHENOTYPIC COVARIANCE}

105 hyperellipse in the original variable space. Principal components analysis moves this

106 hyperellipse to the origin, rotates it, and defines its principal axes. VanValen realized that

107 this hyperellipse had two salient properties: 1) its dimensionality, and 2) its variance, or

108 dispersion, on each axis, which he called 'tightness'. In subsequent work the dispersion

109 of the eigenvalues of a phenotypic covariance matrix - this tightness — has received a

110 great deal of attention, while its dimensionality has not (reviewed in Najarzadeh, 2019).

111 Cheverud (1983) introduced the first modern measure of trait integration, defining it as

$$
I=1-\left(\prod_{i=1}^{n} \lambda_{i}\right)^{1 / n}
$$

114 or the geometric mean of the eigenvalues of the correlation matrix subtracted from unity.

115 This quantity is identical to one minus the $n$th root of the generalized variance (Wilks,

116 1932) proposed by VanValen in 1974. In a wider statistical context this quantity is

117 termed the 'standardized generalized variance', or SGV (SenGupta, 1987). The SGV is

118 the geometric mean of the eigenvalue variance, and so may be thought of as the mean

119 diameter of the axes in the hyperellipse. Both Cheverud (1983) and SenGupta (1987)

120 state that the SGV is comparable among spaces of different dimensionality, and this

121 assumption is widely accepted, although it has never been tested (e.g. Najarzadeh, 2019).

122 While both the Cheverud integration and the equivalent SVG have been used as measures

123 of dispersion, a consensus has emerged that the standard deviation of the eigenvalues is a

124 better measure statistically (Pavlicev et al., 2009). Several other metrics have been

125 proposed for quantifying the dispersion of phenotypic matrices, summarized by Pavlicev 
F. R. O'Keefe, J. A. Meachen, and P. D. Polly

126 et al., 2009, while the sample size requirements of various metrics are evaluated by

127 Grabowski and Porto, 2017.

128 This paper explores the performance of the eigenvalue dispersion and the SGV in

129 rank-deficient geometric morphometric matrices, and we define these metrics here.

130 Eigenvalue dispersion is measured as the standard deviation of the eigenvalue vector of

131 the correlation matrix of GPA landmarks, standardized to the mean eigenvector

132 (Goswami and Polly, 2010, modified from Equations 7 and 8):

133

$$
\lambda \sigma=\frac{\sqrt{\frac{\sum_{i=1}^{n}\left(\frac{\lambda_{i}}{\mu \lambda}-1\right)^{2}}{n}}}{\sqrt{n-1}} \quad \text { Eq. } 1
$$

135 In geometric morphometric data sets, either four (2D) or five(3D) degrees of

136 freedom are lost to the Procrustes analysis, so the number of non-zero eigenvalues will be

137 reduced by this amount (Zelditch et al., 2012). This will also be true of the SGV,

138 calculated as the $\mathrm{n}^{\text {th }}$ root of the first $\mathrm{n}-4$ eigenvectors of the correlation matrix. Because

139 we use two-dimensional data sets with 14 landmarks in this paper, the correct formulation

140 of the SVG is:

141

$$
S G V_{24}=\sqrt[24]{\prod_{i=1}^{24} \lambda_{i}} \quad \text { Eq. } 2
$$

\section{Integration and Modularity}




\section{RANK DEFICIENCY IN PHENOTYPIC COVARIANCE}

144 "However, if the structure of a dataset is strongly modular, with several different groups

145 of strongly co-varying traits, then variance will be distributed more evenly across a

146 number of principal components, and eigenvalue variance will be relatively low. Thus,

147 the dispersion of eigenvalues provides a simple measure for comparison of the relative

148 integration or modularity of the structures described in a matrix."-Goswami and Polly,

$149 \quad 2010$, p. 226.

150 In the $21^{\text {st }}$ century the study of GPA covariance matrices has grown to include the field of

151 modularity and integration (Goswami et al. 2014; Klingenberg, 2013). This conception of

152 integration is similar to Van Valen's and Cheverud's in that it studies covariance

153 structure, but differs in attempting to identify variable subsets that covary relative to

154 others. Rather than relying on a single overall measure of dispersion, modularity studies

155 test specific models of landmark covariation against a null model in an attempt to

156 characterize subsets with high intraset connectivity and low interset connectivity

157 (Goswami and Polly, 2010). Because these sets are primary covariance structures in the

158 data, they will exist on the first several principal components, and the entire eigenvalue

159 distribution is not directly relevant. This approach to modularity has yielded powerful

160 hypotheses of evolutionary plasticity and constraint linking development to phenotype,

161 and to evolutionary changes in modularity over deep time (Goswami et al., 2015).

162 Modularity models were first assessed using the RV coefficient introduced by

163 Klingenberg (2008). This coefficient has been superseded by the similar covariance ratio

164 statistic (CR, Adams, 2016), which is more robust to differences in sample size and data

165 dimensionality. Another common approach to assessing modularity model significance is

166 Partial Least Squares (PLS; Goswami and Polly, 2010); this approach is more involved 
F. R. O’Keefe, J. A. Meachen, and P. D. Polly

167 mathematically but has the same goal as the CR statistic (for a recent example on dog and

168 wolf crania see Curth et al., 2017). As stated in the quote above, the modularity concept

169 can also be applied to a whole shape. In whole-shape modularity, a shape would be more

170 integrated if it had greater eigenvalue dispersion, and would be more modular if it

171 possessed lesser dispersion. This is a specific prediction: in a population where

172 modularity is evolving, an increase in modularity should lead to a decrease in integration

173 and hence dispersion, and thus a decrease in dispersion metrics like SVG and eigenvalue

174 standard deviation.

175

176 Rank Deficiency in Phenotypic Covariance Matrices

177 It [PCA] is used to obtain a more economic description of the $N$-dimensional dispersion

178 of the original data by a smaller number of "principal components", which are formally

179 the eigenvectors of the dispersion matrix... Hence the number of principal components

180 that contribute significantly to the variation of the sample is the actual "dimensionality"

181 of the dispersion. - G. P. Wagner, 1984, pp. 92-93.

182 The rank of a matrix is the dimensionality of the vector space spanned by its variables. If

183 all variables are completely independent then the matrix is of "full rank," equal to the

184 number of variables, but when one variable depends completely on another, the matrix

185 has "deficient rank". The full rank value of a matrix is the number of columns, while the

186 "real rank" is the maximum number of linearly independent columns. The derivation of

187 the covariance or correlation matrix $\mathbf{K}$ directly from $\mathbf{X}$,

$$
K_{v_{i} v_{j}}=\operatorname{cov}\left(x_{i} x_{j}\right)
$$




\section{RANK DEFICIENCY IN PHENOTYPIC COVARIANCE}

190 often results in a covariance matrix that is overdetermined; the high degree of

191 correlation in biological systems often results in matrices whose full rank is much higher

192 than the real rank (Van Valen, 1974; Adams, 2016). Significant rank deficiency in K will

193 impact the eigenvalue distribution, because eigenvalue distributions of rank deficient

194 matrices will have tails of very small non-zero eigenvalues. This tail of small eigenvalues

195 may impact integration metrics that rely on the eigenvalue distribution, but this subject

196 has not been treated explicitly. Characterizing this problem of rank deficiency, and

197 developing treatments for it, are the goals of this paper.

198 In geometric morphometric covariance matrices, rank deficiency can arise from at

199 least three sources. These are the Generalized Procrustes Analysis itself, lack of matrix

200 information, and phenotypic covariance. Of these, only phenotypic covariance is of direct

201 biological interest, while the first two may confound attempts to measure it. The

202 magnitude of rank deficiency due to Procrustes analysis is known; the translation,

203 scaling, and rotation performed during the analysis remove four degrees of freedom from

204 two-dimensional data (Zelditch et al., 2012). A covariance matrix derived from $\mathbf{X}$ should

205 therefore possess $v-4$ non-zero eigenvalues, or in other words be deficient by four ranks

206 from the full rank.

207 The rank deficiency resulting from the information intrinsic to $\mathbf{X}$ is harder to

208 quantify. This lack of information can have two sources: insufficient sample size and

209 landmark oversampling. Lack of information due to sample size arises from too few

210 sampled specimens. Grabowski and Porto (2017) studied the sample size requirements

211 for a range of integration metrics and concluded that an $n$ of greater than 100 was 
F. R. O'Keefe, J. A. Meachen, and P. D. Polly

212 required to achieve values stable to changes in $n$. Integration studies with sample sizes of

213 less than 100 are therefore information poor, and this should increase rank deficiency.

214 What we term landmark oversampling refers to the dense landmarking of a relatively

215 simple shape. A simple shape with few modes of variation requires few landmarks to

216 characterize fully (a circle requires one landmark). Use of more than the necessary

217 number of landmarks will result in covariance among the variables, and increased rank

218 deficiency. This source of rank deficiency has also not been quantified. However it is

219 tractable, as we outline in the Discussion.

220 Another factor impacting matrix rank is use of the correlation matrix rather than

221 the covariance matrix. Use of the correlation matrix artificially inflates matrix rank,

222 because each coordinate of each variable is awarded a full rank. This tacitly assumes that

223 the coordinates within a landmark are uncorrelated, and that variance magnitudes in each

224 coordinate direction are equivalent. However landmark displacements that are dominated

225 by a vector parallel to one axis will have trivial variation on the other, and in a correlation

226 matrix this trivial variation will be awarded a full rank and expanded to a variance of one.

227 A correlation matrix of $\mathbf{X}$ will therefore possess a degree of noise from inflation of trivial

228 variance (for discussion of the desirability of the covariance matrix in morphometric

229 applications see Goswami and Polly, 2010, p. 217). Therefore use of the correlation

230 matrix is unwise with morphometric data unless some procedure is used to concatenate

231 the landmark coordinates into a single measure before analysis (Goswami and Polly,

232 2010, and previous authors have used the congruence coefficient, although this has been

233 criticized by Klingenberg 2008). 


\section{RANK DEFICIENCY IN PHENOTYPIC COVARIANCE}

\section{Information Entropy and Effective Rank}

236 The first to point out the problem of redundancy in the full and real ranks of $\mathbf{K}$ in a

237 morphometric context was Van Valen (1974), who tailored an information metric to

238 correct for it. The problem of rank deficiency can be framed in terms of the eigenvalues

239 of a matrix, with the true rank represented by the subset of eigenvalues carrying

240 significant variance. This differs from the set of eigenvalues that carry non-zero variance,

241 which is how matrix rank is usually defined. The question of the number of 'significant'

242 eigenvalues is a general question in data analysis and there are many techniques for

243 determining this number, summarized by Cangelosi and Goriely (2007) in the context of

244 cDNA microarray data. They include the broken stick model, Cattell's SCREE test, and

245 Bartlett's sphericity test among many others (see also Hine and Blows, 2006; Bunea et

246 al., 2011). All these techniques share fundamental shortcomings, such as an integer value

247 and recourse to an ad hoc criterion to assess the cutoff for significance. Yet the problem

248 of redundancy in eigenvalue distributions is general, and has been solved in signal

249 processing contexts by the use of "information entropy" (Shannon, 1948). Information

250 entropy is a metric used to represent the information content of a system by determining

251 how much a signal can be compressed without loss of information. At least two metrics

252 based on Shannon's information entropy have been proposed to characterize the

253 dimensionality of covariance matrices in a biological context (for a thorough

254 mathematical development see Cangelosi and Goriely 2007). Those authors develop a

255 dimensionality metric for cDNA microarray data they termed the 'information

256 dimension'. A very similar metric was developed by Roy and Vetterli (2007); those

257 authors term their metric the 'effective rank', and use it synonymously with 'effective 
F. R. O'Keefe, J. A. Meachen, and P. D. Polly

258 dimensionality'. The following application of the Shannon entropy to geometric

259 morphometrics follows the Roy and Vetterli (2007) development of effective rank.

$260 \quad$ Given a GPA covariance matrix $\mathbf{K}$ with eigenvalues $\Lambda_{\mathbf{V}}$ :

$$
\lambda_{1} \geq \lambda_{2} \geq \cdots \geq \lambda_{v} \geq 0
$$

$$
E_{s}=-1 * \sum_{i=1}^{v}\left(\frac{\lambda_{i}}{\sum \Lambda_{V}}\right)\left(\ln \frac{\lambda_{i}}{\sum \Lambda_{V}}\right) \quad E q .3
$$

267 because the information entropy was originally defined in a probability context, and the

268 terms being evaluated must therefore sum to unity (Shannon, 1948). Roy and Vetterli

269 define their effective rank as $e$ raised to the power $E_{s}$; the 'effective rank' of $\mathbf{K}$ is defined

270 in the same way:

$$
R_{e}=e^{E s} \quad E q .4
$$

273 This effective rank, $R_{e}$, of $\mathbf{K}$ is a continuous metric, is based on solid theoretical

274 grounds from information theory, and may be thought of as the signficant number of

275 dimensions of the shape space represented by $\mathbf{K}$, or equivalently as the numner of non-

276 redundant eigenvalues in $\mathbf{K}$. 


\section{RANK DEFICIENCY IN PHENOTYPIC COVARIANCE}

278 Study Design

279 This paper has three main components, with the later dependent on the results of the

280 earlier. Therefore we divide the Materials and Methods section into an independent

281 Materials section, followed by three sections of Methods and Results. This organization

282 allows sequential presentation of results as they become necessary for further

283 methodological development. The following sections are:

Materials-This section introduces the three data sets used in this paper. These

285 comprise two samples of dire wolf jaws from Rancho La Brea (RLB), originating from

286 tar pits deposited about 5000 years apart. The third data set consists of jaws from the

287 sabertooth cat, Smilodon fatalis, assembled from multiple RLB pits $(n=81)$. All three

288 data sets comprise 14 two-dimensional landmarks taken from photographs of jaws in

289 lateral view.

290 Methods and Results, Part 1-In this section, we use the concept of effective rank

291 to investigate the rank deficiency of the Pit 61/67 dire wolf population. We demonstrate

292 that the covariance matrix based on these data is highly rank deficient. Through use of a

293 permutation test, we characterize the magnitude of the rank deficiency arising from lack

294 of matrix information. When combined with the rank deficiency expected from the GPA,

295 this allows us to quantify the rank deficiency due to phenotypic covariance. Lastly, we

296 quantify the rank inflation arising from use of the correlation matrix.

Methods and Results, Part 2-Here we evaluate the impact of the observed rank

298 deficiency on whole shape integration metrics. We do this by introducing the Pit 13 dire

299 wolf sample, and demonstrating that it is more integrated than the Pit 61/67 sample. This 
F. R. O'Keefe, J. A. Meachen, and P. D. Polly

300 requires testing each population for candidate modularity models, and demonstrating that

301 Pit 61/67 wolves are significantly modular (best described by a two-parameter model),

302 while the Pit 13 wolves are more integrated (best described by a one-parameter model).

303 Current whole shape integration metrics fail to capture this change in integration;

304 therefore we develop a modified version of the SVG based on the effective rank. Because

305 the Pit 61/67 sample size is larger than Pit 13, we can jackknife the Pit 61/67 sample size

306 down to that of Pit 13. This jackknife yields both a confidence interval for hypothesis

307 testing, and normalizes the rank degeneracy due to matrix information content, allowing

308 us to demonstrate that the new metric is successful in capturing the increase in integration

309 in Pit 13 wolves. We term this metric the 'effective dispersion'.

$310 \quad$ Methods and Results, Part 3-In this section, we generalize the concept of

311 effective dispersion to the case of arbitrary matrix information content. The general

312 metric developed here, termed the 'relative dispersion', allows comparison of whole

313 shape integration among data sets of different sample sizes. It also allows for comparison

314 among data sets of arbitrary landmark number, because it is normalized to the

315 information content of the input variables. This normalization relies on the computation

316 of the effective rank of a permuted data matrix. We demonstrate the utility of the relative

317 dispersion metric by analyzing the Smilodon data set, finding that the Smilodon jaw is

318 much more tightly integrated than that of Canis dirus.

319 The last analytical section is followed by a standard Discussion. One topic

320 considered is the extension of the relative dispersion into a dense semilandmark context,

321 allowing quantification of the information content of a comnplete shape. We propose the

322 term 'latent dispersion' for this quantity. 


\section{RANK DEFICIENCY IN PHENOTYPIC COVARIANCE}

\section{MATERIALS}

\section{Background}

326 The primary data in this paper come from two populations of dire wolves (Canis dirus,

327 Figure 1) from the Rancho La Brea tar pit lagerstätten, deposited in what is now Los

328 Angeles during the terminal Pleistocene (Stock and Harris, 1992). The populations come

329 from two pit deposits, Pits 13 and 61/67. The Pit 13 wolves show elevated tooth breakage

330 and wear, while those from 61/67 do not (Binder et al., 2002). Elevated tooth breakage

331 and wear are thought to indicate nutrient stress, as animals both process carcasses more

332 completely, and compete more intensely, when prey resources are scarce (Van

333 Valkenburgh, 1988, 2009; Meloro, 2012). Subsequent work established this pattern

334 beyond doubt and was able to assign approximate ages to the relevant pit deposits

335 (Binder and Van Valkenburgh, 2010; O’Keefe et al., 2014; Brannick et al., 2015).

336 Phenotypic differences between pits have also been documented; morphometric analyses

337 of dire wolf jaws (Brannick et al., 2015) and crania (O'Keefe et al., 2014) detected clear

338 phenotypic differences in Pit 13 wolves relative to those in 61/67. Pit 13 wolves are

339 significantly smaller in overall body size, and also show significant shape differences,

340 including diminution of the viscerocranium relative to the neurocranium. The observed

341 size and shape changes were attributed to a chronic lack of nutrition during development,

342 producing a neotenic adult phenotype in Pit 13. The biological drivers behind this

343 evolutionary change are not relevant here; what is relevant is that there is a significant

344 modularity shift between the two pits. This creates a useful model system comprising two 
F. R. O'Keefe, J. A. Meachen, and P. D. Polly

345 populations of the same species deposited in the same place, separated only in time, and

346 possessing a significant change in modularity.

\section{Data Collection}

349 Fossils utilized in this paper are left dentaries of Canis dirus, from Pits 13 and

350 61/67, housed in the Tar Pit Museum at Hancock Park, Los Angeles. The input data for

351 all analyses were 14 landmarks taken from 119 dire wolf jaws, a subset of the 16

352 landmark data set from Brannick et al. (2015). One hundred and nineteen adult Canis

353 dirus dentaries were included in this study. The labial side of specimens from Pits 13 (n

$354=36)$, and 61/67 $(\mathrm{n}=83)$ were photographed using a tripod-mounted Cannon EOS 30D

355 8.20-megapixel camera. All specimens were anatomical lefts and were laid flat and

356 photographed with a $5 \mathrm{~cm}$ scale-bar. While camera angle and distance were held constant

357 when photographing, scale-bars were used to properly size specimen images in Adobe

358 Photoshop CS2 v.9.0 before landmark digitization; scaling was mostly unnecessary, and

359 always minor.

360 Fourteen homologous landmarks were digitized on each specimen using the

361 program tpsDig2 (Rohlf, 2013). Positions of landmarks were chosen to give a general

362 outline of the mandible and capture information of functional relevance (Brannick et al.,

363 2015; Figure 1). Landmarks on the tooth row were placed on the alveolus so specimens

364 with missing teeth could be included. However, presence of the lower carnassial tooth

365 was required in order to obtain landmark 5. Two landmarks from Brannick et al. were not

366 used; one of these, at the angle of the mandible, was difficult to identify and showed

367 unacceptably large variance relative to the other landmarks. The second was an interior 


\section{RANK DEFICIENCY IN PHENOTYPIC COVARIANCE}

368 landmark in the masseteric fossa; it was deleted to make the dataset amenable to outline-

369 based iterative semilandmarking.

370 Lastly, as a demonstration of the utility of the relative dispersion metric in Part 3,

371 we analyze a data set of Smilodon fatalis jaws (Meachen et al., 2014), again from Rancho

372 La Brea. The data set comprised 14 landmarks digitized from photographs of 81

373 Smilodon jaws originating from Pits 61/67 and 13 at the Tar Pit Museum, and the UCMP

374 collection. Pooling of the Smilodon sample was necessary to increase sample size.

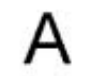

$50 \mathrm{~mm}$

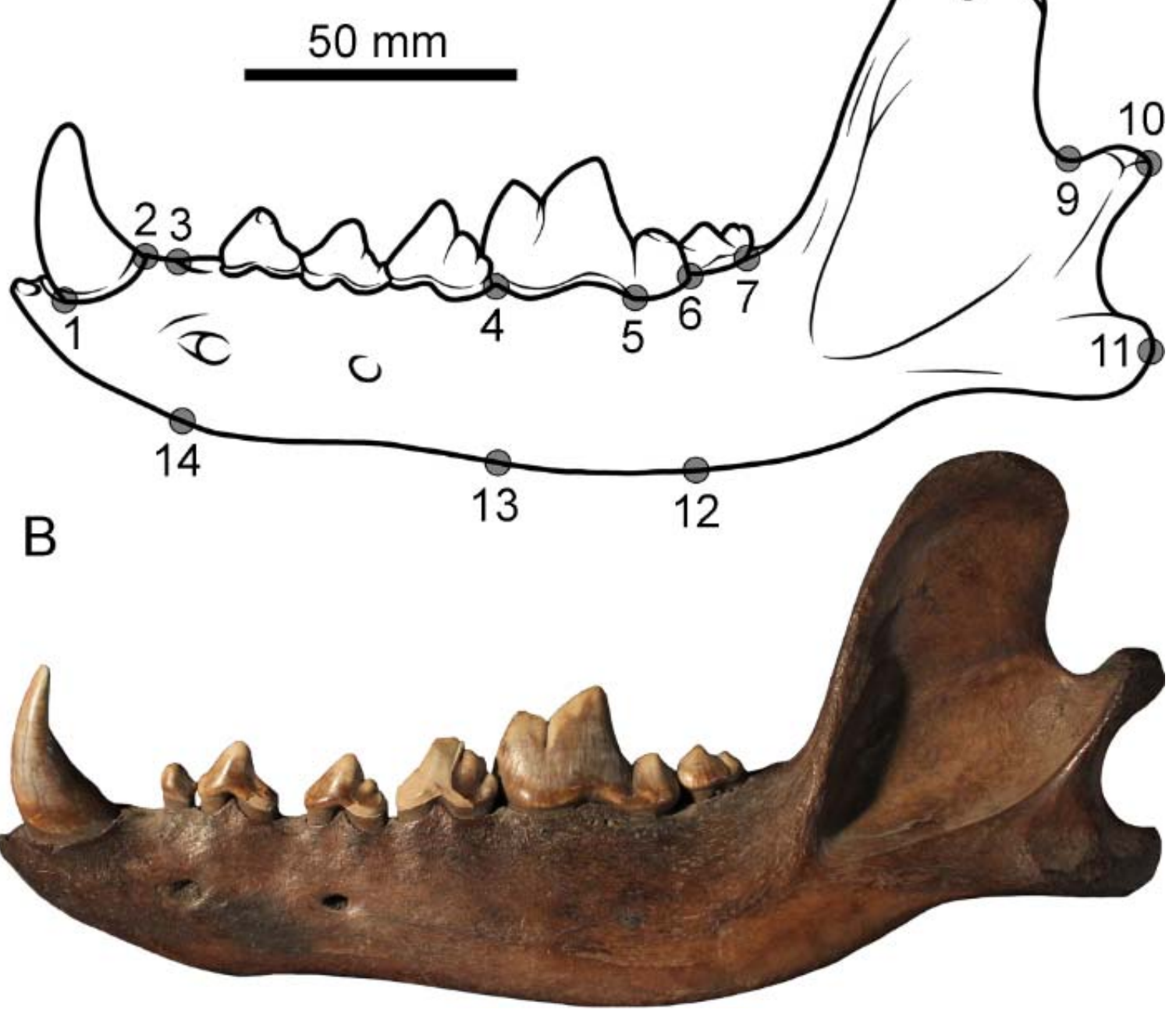


F. R. O’Keefe, J. A. Meachen, and P. D. Polly

Figure 1. Landmarks used in this study, numbered on a schematic dire wolf jaw

378 (A), and (B) a representative Canis dirus dentary from Pit 61/67, Rancho La Brea,

379 California.

380

381 MATERIALS AND RESULTS

382 Part 1: Rank Deficiency in Pit 61/67

383 In this section, we examine the amount of rank deficiency of covariance matrices derived

384 from the Pit 61/67 Canis dirus data set, and quantify where it is coming from. Standard

385 Procrustes superimposition of the landmark data was first performed using the geomorph

386 package (Adams et al., 2020) in R (R Core Team, 2014). Procrustes superimposition

387 yielded a matrix of transformed (GPA) landmarks $\mathbf{X}$ and a vector of centroid size.

388 Principal components analysis of $\mathbf{X}$ was performed on four covariance matrices. These

389 were the correlation and covariance matrices for the original data, and correlation and

390 covariance matrices for permuted data. The scree plots for the eigenvalue vectors of the

391 four matrices are shown in Figure 2. Also shown in Figure 2 are the effective ranks of the

392 four matrices, calculated as defined in Equations 3 and 4. Code for computing the

393 effective rank of covariance and correlation matrices, including the permutation code,

394 was written in R (R Core Team, 2014; see Appendix 1). The effective ranks calculated

395 from the correlation and covariance matrices derived from the real data are unique values.

396 Effective rank was also calculated for 10000 matrices in which each column was

397 permuted; this permuted mean is plotted and the standard deviations are reported in the

398 caption to Figure 2.

399 


\section{RANK DEFICIENCY IN PHENOTYPIC COVARIANCE}

400

401

402

403

404

405

406

407

408

409

410

411

412

413

414

415

416

417

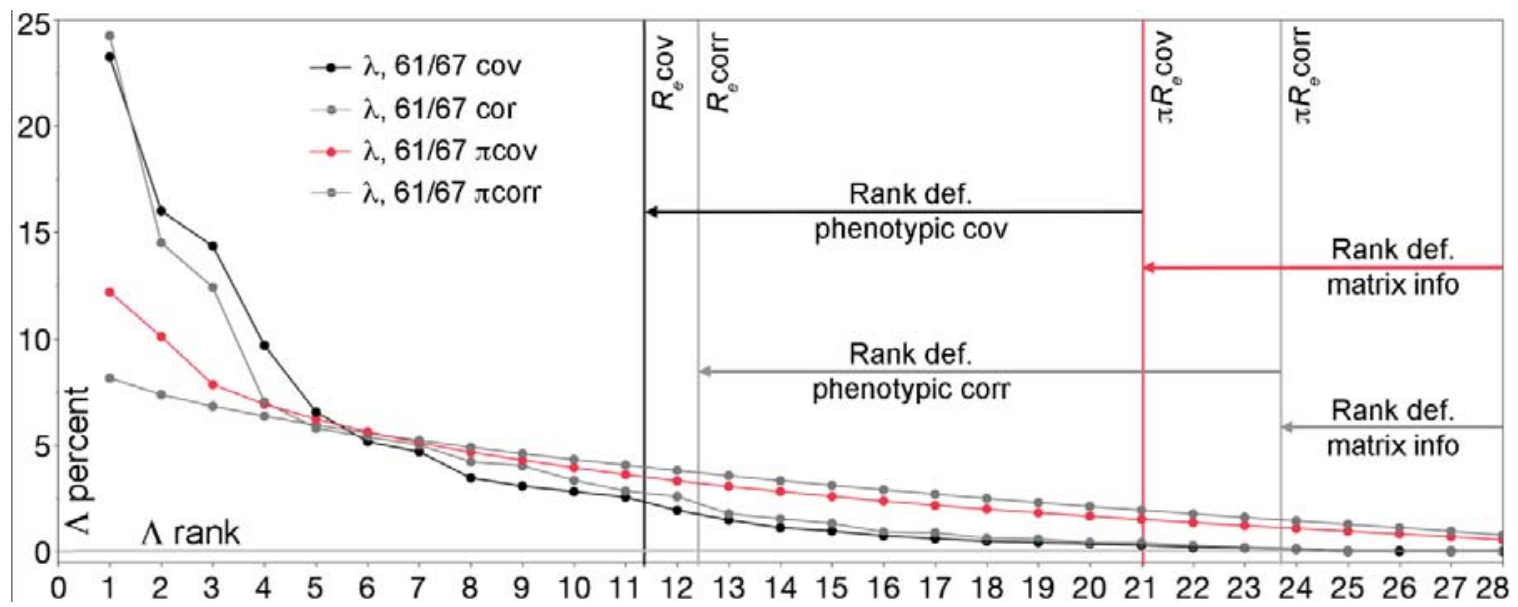

Figure 2. Rank deficiency and its sources in Pit 61/67 dire wolves. Scree plots for

four data treatments are plotted. Note that the last four eigenvalues are exactly zero for

both the correlation and covariance matrices computed from the real data, reflecting the

loss of four degrees of freedom to the GPA. The effective rank of permuted data shows

rank deficiency due to lack of matrix information; the true effective ranks are much

smaller than this, and this additional deficiency is due to phenotypic covariance. Effective ranks for each matrix are: Permuted covariance $=21.028+/-0.226$; permuted correlation $=23.728+/-0.258 ;$ covariance $=11.362 ;$ correlation $=12.424$.

The scree plots of both the correlation and covariance matrices of $\mathrm{X}$ demonstrate

that the vector of eigenvalues for each matrix contains 24 non-zero eigenvalues, the

expected number given the loss of four ranks to the GPA. Eigenvalues 25-28 are non-

zero in the permuted matrices, showing that this rank deficiency is recovered when the

matrix is randomized. Effective rank calculations show that both matrices are also highly

rank deficient. The rank of the covariance matrix is 11.362 , meaning that less than half of

the 24 non-zero eigenvalues carry meaningful information. The number of ranks in the

permuted covariance matrix is about 21 , indicating that the covariance matrix is 


\section{F. R. O’Keefe, J. A. Meachen, and P. D. Polly}

418 information poor by about seven ranks. Four of these are from the GPA; the rest are

419 probably due to insufficient sample size (Grabowski and Porto, 2017). The rank

420 deficiency due to phenotypic covariance is therefore 21 minus the effective rank of the

421 real matrix (11.36), or about 9.64 ranks. Therefore 12.64 ranks, or over half, of the

422 eigenvalue distribution is redundant. The small values of the higher eigenvalues are

423 caused mainly by phenotypic covariance, but they do not carry information about it, and

424 lack of matrix information acerbates this deficiency. The eigenvalue vector is

425 overdetermined with respect to its information, and its distribution will be affected as a

426 result.

427 Figure 2 also shows that the rank of the correlation matrix is 12.424 . Use of the

428 correlation matrix therefore adds over one full rank of information relative to the

429 covariance matrix. We believe this rank increase is noise, as described in the

430 Introduction. The pattern for the permuted matrices is similar; the correlation matrix adds

431 almost three ranks of noise over the value for the covariance matrix.

433 Part 2: Modularity Models and Whole Shape Integration Metrics

434 In this section, we introduce the Pit 13 dire wolves and show they are more integrated

435 than those from Pit $61 / 67$. We do this by testing both populations for modularity against a

436 range of candidate two- and three parameter models. We then calculate the eigenvalue

437 distribution and SVG for the two covariance matrices, and show that both fail to capture

438 the modularity change. We then modify the SVG to use only the non-redundant

439 eigenvalues, and show that this measure does capture the modularity change. 


\section{RANK DEFICIENCY IN PHENOTYPIC COVARIANCE}

441 of the models to be tested. In this paper we use a heuristic approach: examining the

442 multivariate allometry vectors of interlandmark distances to identify candidate models.

443 While important, the specification of models is tangential to our discussion of rank

444 deficiency, and we include it below as Appendix 2. This allows us to move directly to

445 testing the candidate models for the two data sets. A total of 14 models of modularity

446 were suggested by the multivariate allometry analyses of the interlandmark distances, and

447 these models were tested individually using the CR statistic (Goswami and Polly, 2010;

448 Figure 3; Table 1), performed in the geomorph package in R (Adams et al., 2020). The

449 models were tested both on the pooled data, and on data divided by pit. Effect-size tests

450 and tests against a model of zero modules were performed on 61/67 wolves only, as only

451 these wolves displayed significant modularity; representative tests and results are listed in 452 Table 1.

\begin{tabular}{|c|c|c|c|c|c|c|c|}
\hline $\begin{array}{l}\text { Model, vs. all } \\
\text { other LMs }\end{array}$ & $\mathrm{CR}_{\text {pooled }}$ & $P$ value & $\mathrm{CR}_{13}$ & P value & $\mathrm{CR}_{61 / 67}$ & P value & $\mathbf{P}_{\text {null }}$ \\
\hline 1,2 & 1.17 & 0.427 & 1.234 & 0.581 & 1.063 & 0.219 & - \\
\hline $1,2,9,10$ & 1.10 & 0.573 & 1.201 & 0.838 & 1.090 & 0.491 & - \\
\hline $1-7$ & 1.15 & 0.837 & 1.147 & 0.786 & 1.171 & 0.921 & - \\
\hline $3-7$ & 0.96 & $0.052 \sim$ & 1.067 & 0.387 & 0.898 & $0.022 *$ & $0.031 *$ \\
\hline $4-7$ & 0.92 & $0.046^{*}$ & 0.994 & 0.108 & 0.894 & $0.028 *$ & $0.046^{*}$ \\
\hline $\begin{array}{l}1,2,9,10 \\
3,4,5,6,7\end{array}$ & 1.06 & 0.244 & 1.192 & 0.719 & 1.029 & 0.226 & - \\
\hline
\end{tabular}


F. R. O'Keefe, J. A. Meachen, and P. D. Polly

Table 1. Results of representative modularity model tests. Representative module

454 models are listed on the left column, with CR statistics for three data partitions in

455 subsequent columns. Pit 61/67 wolves have two significant modules comprising the

456 check teeth vs. the rest of the jaw, while Pit 13 wolves do not show significant modularity

457 between these or any other modules. As shown in Figure 3, Pit 13 wolves are best fit with

458 a one-parameter model, while Pit 61/67 wolves are best fit with a two-parameter model.

459 Wolves from 61/67 are therefore significantly more modular than those from Pit 13.

460 Other modularity models tested that were not significant include the two-parameter

461 models $(4,5,6,7,13,14),(4,5,6,7,8),(4,5,6,7,8,13,14),(1,2,8,9,10)$, and $(1,2,14,9,10,11)$,

462 and the three-parameter models $((1,2,8,9,10)(4567)),((1,2,9,10)(4,5,6,7,13,14))$, and

$463((129,10)(4,5,6,7,8))$.

There are two models with statistically significant CR values (Table 1). Both are

466 two parameter models that contrast the length of the post-canine tooth row with the rest

467 of the jaw. The effect sizes of the modularity models with significant CRs were compared

468 using the compare.CR function in geomorph; the effect sizes were not significantly

469 different. Comparison with a null model of zero modules was performed using the same

470 function. For Pit 61/67 wolves only, the cheek tooth model (3-7) was significantly better

471 than the null model, while the model containing only the molars (4-7) was marginally so.

472 The anterior-posterior width of the canine and of the condyloid process $(1,2,9,10)$ do not

473 form a distinct module on their own; the significant CR of the three-module model is

474 driven by the presence of the cheek tooth module. The cheek teeth are clearly a module.

475 The canine does not take part in this module, and therefore has significant variance 


\section{RANK DEFICIENCY IN PHENOTYPIC COVARIANCE}

476 attributable to another factor. The inability of the modularity tests to identify the canine

477 and condyloid process as a module was surprising. We believe it was not identified

478 because most of its variation covaries with size. The cheek teeth vary against size, and

479 this allows identification of that module. We note that Pit 13 wolves had no significant

480 modules, and must therefore be more integrated than Pit 61/67 wolves.
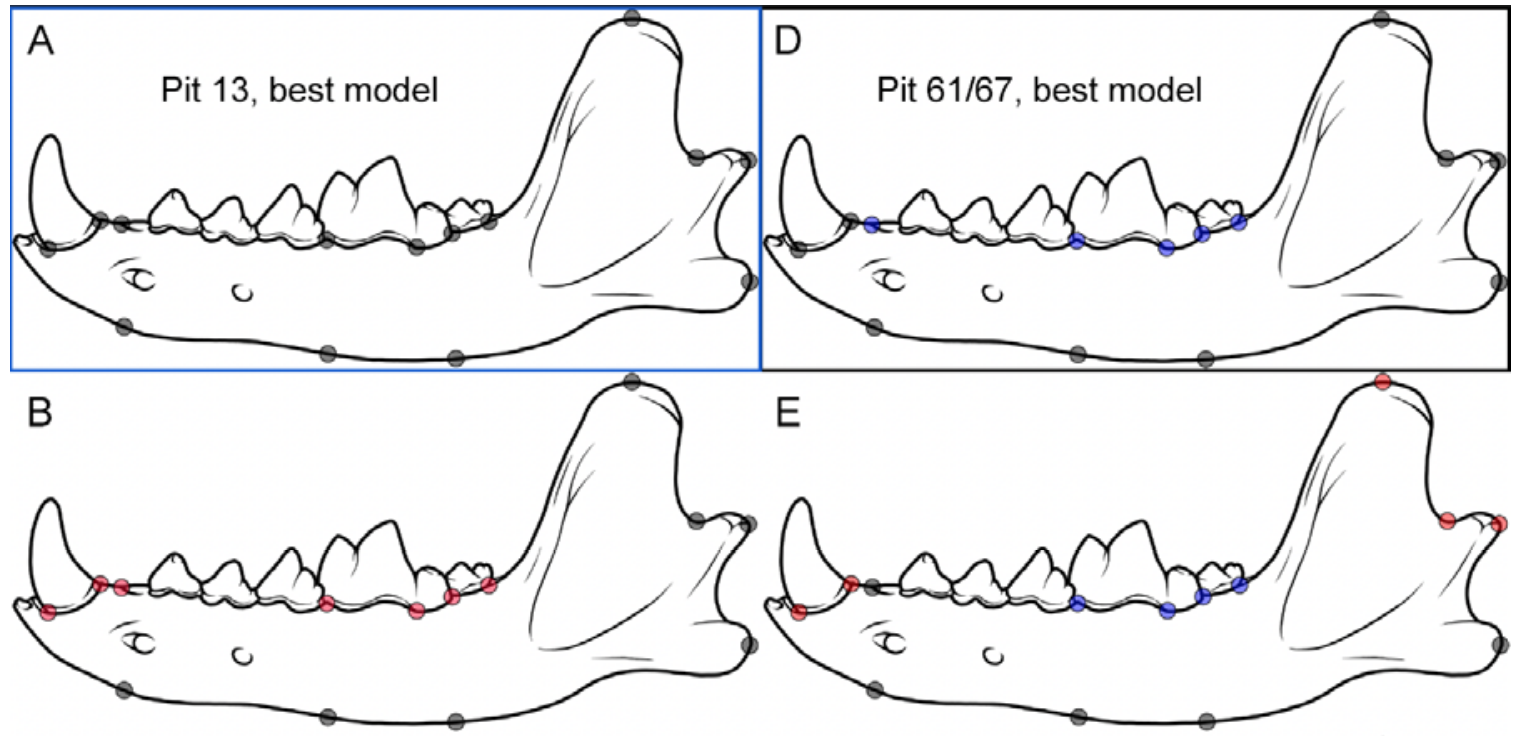

C

483 Figure 3. Candidate modularity models for the Canis dirus jaw tested in this paper. The

484 preferred modularity model for Pit 61/67 wolves is D, a two-parameter model of

$485(3,4,5,6,7)$ vs. all other landmarks. This is the model with the best statistical support, but

486 only in Pit 61/67 (Table 1). The preferred model for Pit 13 wolves is A, the one-

487 parameter model. The other models shown did not have significant modularity according

488 to the CR statistic; all models tested are listed in the caption to Table 1. 
F. R. O'Keefe, J. A. Meachen, and P. D. Polly

The magnitude of the CR differences between pits was surprising, given they are

491 taken from populations of the same species at the same location, sampled 5000 years

492 apart. Given that the sample size of Pit 13 was 36 while that of $61 / 67$ was 83 , we were

493 concerned that a lack of statistical power was obscuring the modularity signal in Pit 13.

494 To test for this, we jackknifed the 61/67 sample to 36 members to mirror the sample size

495 of Pit 13 before re-running the CR analysis. Although confidence intervals widened in

496 these subsets, the covariance structure of Pit 61/67 was still clearly evident and

497 significantly modular, indicating that the modularity difference between pits is not

498 attributable to difference in sample size. The pooled CR statistics, run on all 119 wolves,

499 indicate that the inclusion of the Pit 13 wolves actually degrades the global modularity

500 signal (Table 1). This indicates that the relative lack of modularity in Pit 13 is real.

Measures of Whole-Jaw Integration - the eigenvalue dispersion and SVG metrics

503 were calculated using eigenanalysis, bootstrapping, and permutation codes written in $\mathrm{R}$

504 (R Core Team, 2014; Appendix 1). The eigenvalue standard deviation metric is

505 significantly less in Pit 13 wolves (Table $2: \lambda \sigma, 13<61 / 67, p=0.00008$ ), while the

$506 \mathrm{SGV}_{24}$ metric is equivalent between the two groups $(13=61 / 67, p=0.837$, Student's $T$ in

507 this and subsequent comparisons). This implies that eigenvalue dispersion is significantly

508 less in Pit 13, and those wolves should be more modular than Pit 61/67. Yet this is not the

509 case; the modularity tests reported above clearly show that Pit 61/67 wolves are

510 significantly modular, while Pit 13 wolves are not. The eigenvalue dispersion is less in

511 Pit 13 even though the jaws are more integrated. The $\mathrm{SGV}_{24}$ metric fails to detect the 


\section{RANK DEFICIENCY IN PHENOTYPIC COVARIANCE}

512 increase in modularity. As demonstrated above, over half of the ranks used to calculate

513 both metrics are redundant, and we believe the $\mathrm{SGV}_{24}$ is essentially measuring noise. We

514 experimented with the use of the covariance matrix in the $\mathrm{SGV}_{24}$ to see if the failure of

515 that metric was due to use of the correlation matrix. Use of the covariance matrix

516 required standardization of the eigenvalues before calculation to control for different

517 matrix variances. This modified metric is also reported in Table 2, and like the $\mathrm{SGV}_{24}$ it

518 is equivalent between the two matrices. Rank inflation due to use of the correlation

519 matrix is therefore not the reason for failure of the $\mathrm{SGV}_{24}$.

522 rank for the two populations indicates that the rank of Pit 61/67 is less than that of Pit 13

523 (Figure 4: $R_{e}, 13>61 / 67, p=0.00148$ ). To account for this difference in dimensionality

524 dispersion, we modify the SGV to consider only the non-redundant eigenvalues of the

525 covariance matrix. This metric includes only the eigenvalues that carry significant

526 information, i.e. those up to the effective rank of the matrix. We label this quantity

$527 \mathrm{SGV}_{\mathrm{Re}}$, and calculate it as follows:

528

$$
S G V_{R e}=\sqrt[R e]{\prod \lambda_{R e}}=\sqrt[R e]{\left(\prod_{i=1}^{[R e]} \lambda_{i}\right) *\left(R_{e}-\left[R_{e}\right]\right)\left(\lambda_{[R e]+1}\right)} \quad E q .5
$$

530 Where $R_{e}$ is the effective rank of the covariance matrix, and $\left[R_{e}\right]$ is the integer value of

531 the effective rank. The $\mathrm{SGV}_{\mathrm{Re}}$ is the $R_{e}^{\text {th }}$ root of the product of the eigenvalues up to the

532 integer value of $R_{e}$, multiplied by the decimal remainder of $R_{e}$ times the next smallest 
F. R. O'Keefe, J. A. Meachen, and P. D. Polly

533 eigenvalue. The value for this statistic was calculated for the different data partitions

534 using code written in R, with jackknifed confidence intervals for Pit 61/67 (Table 2;

535 Appendix 1). Using this measure, Pit 13 wolves have significantly higher dispersion than

536 those of Pit 61/67. Hence they are more integrated, while 61/67 wolves are more

537 modular. The $\mathrm{SGV}_{\mathrm{Re}}$ metric successfully recovers the increase in modularity exhibited by

$53861 / 67$ wolves.

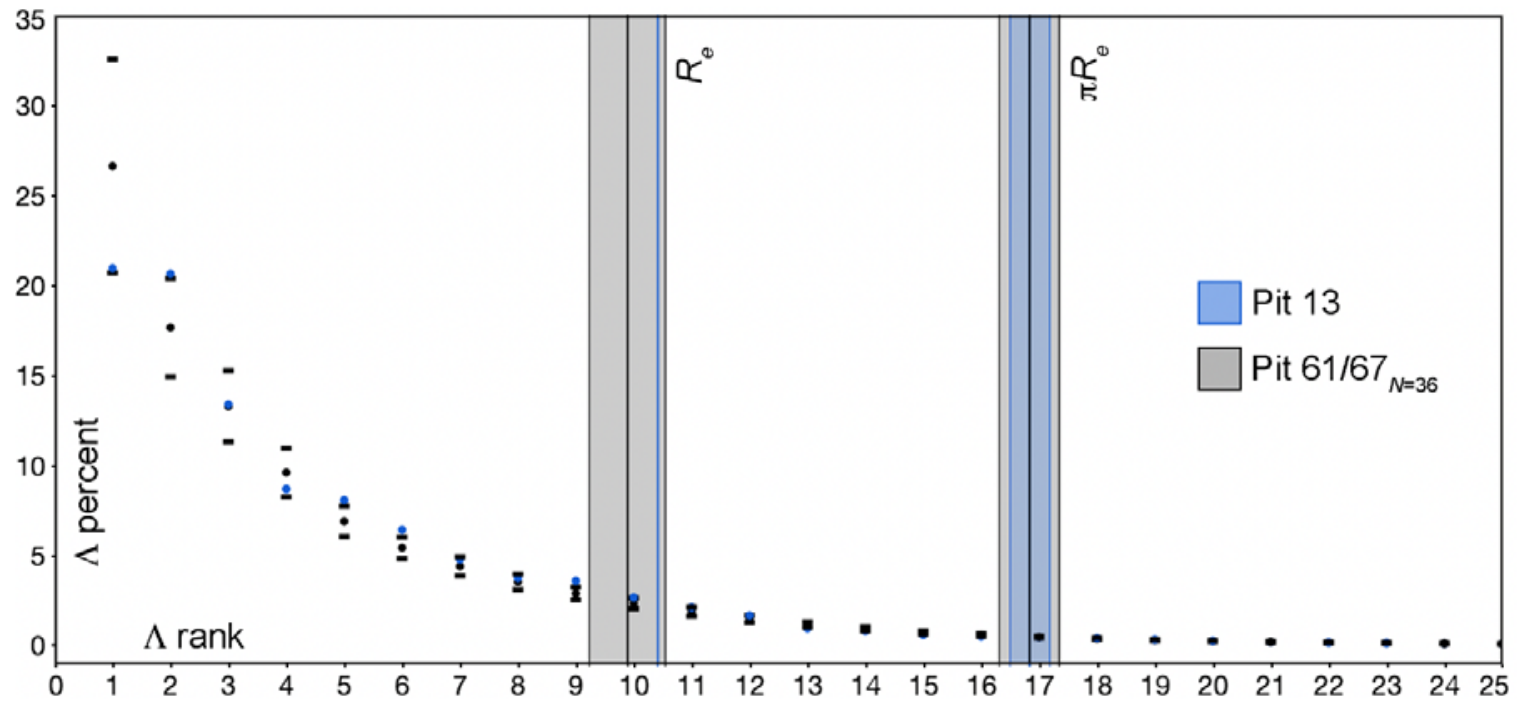

$540 \quad$ Figure 4. Scree plots and effective rank for GPA landmark data of Canis dirus

541 jaws. Blue is Pit 13, black is Pit 61/67. Confidence intervals for effective rank $\left(R_{e}\right)$ are

54210000 jackknife replicates of the Pit 61/67 sample to an $n=36$. Eigenvalue dispersion

543 appears greater in Pit 61/67 wolves, even though they have significant modularity and Pit

54413 wolves do not. However the amount of total variance is greater in Pit 13, and the Pit

54513 effective rank is significantly larger, so the variance is spread over a greater number of

546 dimensions. The permuted dimensionality is also shown on both plots; this value is

547 identical between pits $\left(\pi R_{e}=16.84\right)$, demonstrating that the rank deficiency difference

548 between the pits is due to phenotypic covariance only (i.e. the rank deficiency due to lack

549 of matrix information is equivalent). The effective rank of both matrices is very deficient, 


\section{RANK DEFICIENCY IN PHENOTYPIC COVARIANCE}

550 with fewer than half of the 24 eigenvalues being meaningful. The effective rank of Pit 13

551 wolves is 10.414; the permuted effective rank is $16.841+/-0.345$. The jackknifed

552 effective rank for Pit $61 / 67$ wolves is $9.89+/-0.656$, while the permuted value at $n=36$

553 is $16.832+/-0.519$.

Previous authors rejected SGV as a statistic to measure dispersion because its

556 distribution is not linear (Pavlicev et al., 2009); they prefered the standard deviation of

557 the eigenvalues because of its linearity and because it has the same units as the input data.

558 Because the $\mathrm{SGV}_{\mathrm{Re}}$ is a measure of variance, one may utilize the definition of the

559 standard deviation to transform the $\mathrm{SGV}_{\mathrm{Re}}$ into a similar measure:

560

$$
D_{e}=\sqrt{S G V_{R e}}=\sqrt[2 R e]{\left(\prod_{i=1}^{[R e]} \lambda_{i}\right) *\left(R_{e}-\left[R_{e}\right]\right)\left(\lambda_{[R e]+1}\right)} \quad \text { Eq. } 6
$$

562 where the square root of the $S G V_{R e}$ is a new metric, $D_{e}$, that we call 'effective dispersion'.

563 It measures dispersion in both variance and dimensionality together, and accounts for the

564 rank deficiency that misleads the classical integration metrics. The $D_{e}$ metric is

565 significantly different between the two samples $(p=0.0049$; Table 2).

\begin{tabular}{|c|c|c|c|c|c|}
\hline $\begin{array}{l}\text { Data } \\
\text { Partition }\end{array}$ & $\lambda \sigma,+/-$ & $\begin{array}{l}S G V_{24}, \\
+/-\end{array}$ & $\begin{array}{l}\operatorname{covSG} V_{24}, \\
+/-\end{array}$ & $\begin{array}{l}S G V_{R e} \\
+/-\end{array}$ & $\begin{array}{l}D_{e} \\
+/-\end{array}$ \\
\hline C. dirus 13 & 0.277 & 0.363 & 0.0108 & $4.131 \mathrm{e}-5$ & 0.0064 \\
\hline
\end{tabular}


F. R. O’Keefe, J. A. Meachen, and P. D. Polly

\begin{tabular}{lllllll}
\hline$n=36$ & & & & & \\
\hline C. dirus 6167j & 0.304 & 0.362 & 0.0108 & $3.496 \mathrm{e}-5$ & 0.0058 \\
$n=36$ & 0.027 & 0.029 & 0.0008 & $1.42 \mathrm{e}-5$ & 0.0012 \\
\hline
\end{tabular}

567 Table 2. Whole jaw integration measures and other statistics for the Canis dirus

568 mandible data sets. All reported confidence intervals are one standard deviation of 10000

569 jackknife or permutation replicates. The $D_{e}$ metric is significantly different between the

570 two samples $(p=0.0049)$. Quantities listed are eigenvalue standard deviation (correlation

571 matrix, 24 eigenvalues), $(\lambda \sigma)$; standardized generalized variance $24\left(\boldsymbol{S G} \boldsymbol{V}_{\mathbf{2 4}}\right)$;

572 standardized generalized variance 24 using the covariance matrix, and standardizing the

573 eigenvalue vector to the sum of eigenvalues $\left(\boldsymbol{c o v} S \boldsymbol{G} \boldsymbol{V}_{24}\right)$; standardized generalized

574 variance effective $\operatorname{rank}\left(\boldsymbol{S} \boldsymbol{G} \boldsymbol{V}_{\boldsymbol{R} e}\right)$; and effective dispersion $\left(\boldsymbol{D}_{\boldsymbol{e}}\right)$.

Part 3: Matrix Information and Relative Dispersion

Permuted Rank Standardization - Sample size impacts effective rank; the

578 effective rank of $61 / 67$ wolves at $n=36$ is 9.89 , while the full matrix of $n=83$ has an

579 effective rank of 11.362 (Figures 2 and 4). Clearly the derivation of a version of $D_{e}$ that

580 accounts for matrix size is desirable, as this will allow comparisons between matrices of

581 different sizes. Pavlicev et al. (2009) accomplish a similar standardization for eigenvalue

582 standard deviation by dividing the observed eigenvalue standard deviation by its

583 maximum possible value to yield their "relative standard deviation". Because they use the

584 correlation coefficient in their calculations, the minimum possible correlation in a matrix

585 is simply the number of traits minus one, because each trait adds an additional unit of

586 uncorrelated variance. A relative version of effective dispersion is more difficult to 


\section{RANK DEFICIENCY IN PHENOTYPIC COVARIANCE}

587 calculate because the minimal covariance in a matrix is not derivable from first

588 principles, because the input variance for each variable is not one. Also, because the

589 matrix is rank deficient this must also be considered, suggesting that an approach based

590 on effective rank is necessary. The quantity needed for standardization must therefore

591 preserve the variances of the input variables, but remove their statistical covariance, and

592 must also be treated for rank deficiency. One can generate a quantity with these

593 properties by permuting the columns in $\mathbf{X}$ and then calculating the resulting covariance

594 matrix. This matrix will preserve the input variances on the diagonal, but will remove

595 statistical covariance on the off-diagonal (although covariances will still be non-zero in a

596 rank-deficient matrix). The effective rank of the permuted matrix can then be calculated,

597 yielding $\pi R_{e}$. This permuted effective rank is the maximum matrix rank given the

598 variable input variances and no statistical correlation among them. It is the amount of

599 matrix information, and is driven by sample size in reasonably complex shapes. It can be

600 used as a basis for standardizing $D_{e}$ for matrix size.

601 The appropriate scaling is based on consideration of what the effective dispersion

602 actually is, namely the square root of the geometric mean of a subset of the eigenvalues

603 of $\mathbf{K}$, or alternatively, the root of the variance of one dimension of the hyperellipse.

604 Because $D_{e}$ it is the square root of a mean eigenvalue (a variance), it has the same units as

605 the original data (Pavlicev et al, 2009), and we would like to conserve this property in the

606 scaled coefficient. Therefore there should be no variance term in the standardization on

607 dimensional grounds. More importantly, the other property of $D_{e}$ is that it has been

608 limited to its non-redundant information content, and we wish to scale $D_{e}$ to an

609 expectation of maximum possible information content per axis. We are not concerned 


\section{F. R. O'Keefe, J. A. Meachen, and P. D. Polly}

610 with scaling for the variance in the matrix, but for the amount of information in one

611 dimension of an uncorrelated matrix that conserves this variance. The appropriate scaling

612 term is therefore one rank of the permuted matrix, or $1 / \pi R_{e}$. This yields the definition for

613 the "relative dispersion", or $D_{r}$ :

614

$$
D_{r}=\frac{D_{e}}{\sqrt{\frac{1}{\pi R_{e}}}}=\frac{\sqrt[R e]{\prod \lambda_{R e}}}{\sqrt{\frac{1}{\pi R_{e}}}} \quad \text { Eq. } 7
$$

616 Relative Dispersion of Canis dirus and Smilodon- To demonstrate the use of

617 relative dispersion, we turn to the third data set used in this paper, that of RLB Smilodon

618 fatalis (Meachen et al., 2014). These data comprise 14 two-dimensional landmarks on 81

619 jaws, hence are quite similar to the dire wolf data (11 of the 14 landmarks are

620 homologous between Smilodon and Canis dirus). We began by calculating the effective

621 rank for Smilodon and comparing it to Pit 61/67 dire wolves (Figure 6). Both samples

622 were bootstrapped for confidence intervals, and the sample size for 61/67 was held at 81

623 during the bootstrap. Both procedures lower effective sample size, and so reduce matrix

624 information and hence rank. The exact values for integration statistics are reported in

625 Table 4, along with the bootstrap means and standard deviations. Figure 6 plots the $n=$

62681 bootstrap values for effective rank. 


\section{RANK DEFICIENCY IN PHENOTYPIC COVARIANCE}

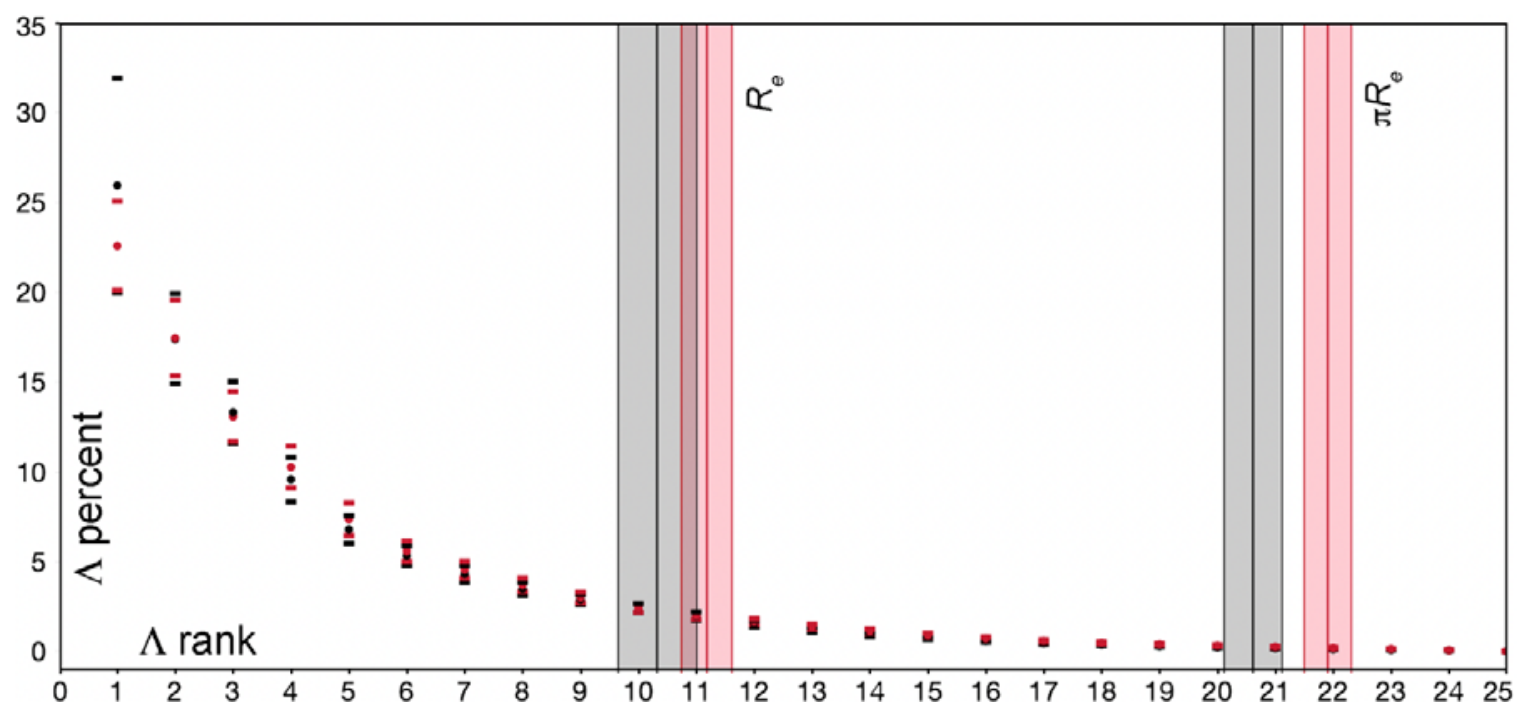

$629 \quad$ Figure 6. Scree plot comparison for Canis dirus and Smilodon data sets, 14

630 landmarks on jaws, at $n=81$. Plotting conventions are the same as those used in Figure 2.

631 Canis dirus 61/67 is shown in black, Smilodon in red. Both data sets have been

632 bootstrapped for a confidence interval (10000 replicates), hence the values shown here

633 are less than the precise values of $R_{e}$ for $C$.dirus (11.36) and Smilodon (12.32; Table 4).

634 Because bootstrapping lowers the effective sample size, this drop in rank illustrates the

635 strong sensitivity of the effective rank calculation to sample size. The plotted means and

636 standard deviations are: Canis dirus 61/67, $R_{e}=10.33+/-0.677 ; \pi R_{e}=20.614+/-0.503$.

637 Smilodon $R_{e}=11.184+/-0.438 ; \pi R_{e}=21.91+/-0.503$.

640 highly overdetermined, although the effective rank of Smilodon is significantly greater.

641 The permuted ranks of both taxa are much closer to 24 at a sample size of 81 compared to

642 Canis dirus Pit 13, at a sample size of 36. This implies that the permuted rank of the

643 matrices should converge toward 24 at large $n$ (probably over 100, in accord with sample

644 size requirements of other integration metrics as shown by Grabowski and Porto, 2017). 


\section{F. R. O’Keefe, J. A. Meachen, and P. D. Polly}

645 The relative dispersion is comparable among data sets of different sample sizes, as

646 demonstrated in Table 4 (the $D_{e}$ of the $61 / 67 n=36$ jackknife sample is 0.0058 , while

647 that of the full $n=83$ data set is 0.0052 ; the $D_{r}$ of both partitions is 0.0238 ). The values in

648 Table 4 demonstrate that relative dispersion functions as intended; it is robust to

649 difference is sample size, and successfully captures the modularity evolution between

650 dire wolf populations. The comparison for $D_{r}$ is marginally significant, while that for

651 sample-size controlled $D_{e}$ was strongly significant (Table 2); the reasons for this loss of

652 power were not investigated. Lastly, the $D_{r}$ metric shows that the Smilodon jaw is much

653 more tightly integrated than that of Canis dirus. We note that the $S G V_{24}$ successfully

654 recovers this difference, while the eigenvalue standard deviation is still incorrect.

\begin{tabular}{llllll}
\hline Data Partition & $\lambda \sigma_{24},+/-$ & $S G V_{24}$ & $S G V_{R e}$ & $D_{e}$ & $D_{r}$ \\
\hline C. dirus $13 n=36$ & 0.277 & 0.363 & $4.131 \mathrm{e}-5$ & 0.0064 & 0.0263 \\
BS estimate & 0.316 & -- & $5.029 \mathrm{e}-5$ & 0.00690 & 0.0283 \\
BS sd & 0.015 & -- & $2.283 \mathrm{e}-5$ & 0.00162 & 0.0066 \\
\hline C. dirus $6167 n=83$ & 0.278 & 0.500 & $2.71 \mathrm{e}-5$ & 0.0052 & 0.0238 \\
BS estimate & 0.296 & 0.421 & $3.373 \mathrm{e}-5$ & 0.00567 & 0.0260 \\
BS sd & 0.025 & 0.030 & $1.311 \mathrm{e}-5$ & 0.00112 & 0.0051 \\
\hline Smilodon $\mathrm{n}=81$ & 0.250 & 0.575 & $7.20 \mathrm{e}-5$ & 0.0085 & 0.0391 \\
BS estimate & 0.270 & 0.480 & $9.098 \mathrm{e}-5$ & 0.00940 & 0.0432 \\
BS sd & 0.010 & 0.024 & $3.055 \mathrm{e}-5$ & 0.00161 & 0.0074 \\
\hline
\end{tabular}

656 Table 3. Whole jaw integration measures for the Canis dirus jaw samples, and for

657 the Smilodon data. The relative dispersion difference between Pit 13 and Pit 61/67 wolves 


\section{RANK DEFICIENCY IN PHENOTYPIC COVARIANCE}

658 is marginally significant (Welch's $T=1.863, p=0.068$ ); Those between Smilodon and

659 the two wolf data sets are highly significant $(13, T=-10.85, p<0.0001 ; 6167, T=-$

$66017.29, p<0.0001)$. The Smilodon jaw is much more tightly integrated than that of Canis

661 dirus. Note that the $S G V_{24}$ also correctly captures the integration difference between

662 Smilodon and Canis dirus, while the eigenvalue standard deviation still fails. Quantities

663 listed are eigenvalue standard deviation (correlation matrix, 24 eigenvalues), $(\lambda \sigma)$;

664 standardized generalized variance $24\left(\boldsymbol{S} G \boldsymbol{V}_{24}\right)$; standardized generalized variance

665 effective rank $\left(\boldsymbol{S} G \boldsymbol{V}_{\boldsymbol{R} e}\right)$; effective dispersion $\left(\boldsymbol{D}_{\boldsymbol{e}}\right)$; and relative dispersion $\left(\boldsymbol{D}_{\boldsymbol{r}}\right)$.

\section{Discussion}

\section{Rank Deficiency and Its Treatment}

669 In this paper we demonstrate that a typical GPA phenotypic covariance matrix is highly

670 rank deficient. This fact is obvious from the scree plot, but quantifying the rank

671 deficiency requires use of the Shannon, or information, entropy. Information entropy is

672 used in signal processing to determine the amount a signal can be compressed without

673 loss of information. We operationalize the Shannon entropy to a geometric morphometric

674 context and use it to calculate that the information, or effective, rank of the dire wolf

675 covariance matrix is less than half of the full rank. Geometric morphometric data sets

676 have several characteristics that make rank deficiency more severe than in a matrix of

677 linear measures. The first is the GPA procedure, which utilizes four degrees of freedom

678 and hence removes four ranks from the covariance matrices dealt with here. This

679 magnitude of this rank loss is known (Figure 1) and can be accommodated. By contrast,

680 The magnitude of rank deficiency due to lack of matrix information is not known. We use 
F. R. O'Keefe, J. A. Meachen, and P. D. Polly

681 a permutation approach to estimate this magnitude, allowing us to calculate the

682 magnitudes of rank deficiency due to phenotypic covariance vs. the other sources.

683 Phenotypic covariance accounts for about 9.6 ranks of deficiency in the dire wolf data set

684 considered in Part 1, while lack of matrix information accounts for about 3 ranks, and the

685 GPA accounts for 4 ranks. This non-phenotypic rank deficiency is worrisome, because

686 misleads whole shape integration metrics that rely on the entire eigenvalue distribution.

687 To demonstrate the impact of rank deficiency on current integration metrics, we

688 show that current whole shape integration metrics fail to identify the modularity change

689 between dire wolf populations. Granted this is a minor change, from a one parameter

690 model to a two parameter model, and the differences in whole-shape metric magnitudes

691 are not large. However eigenvalue standard deviation is statistically significant in the

692 wrong direction, while the $S G V_{24}$ seems to lack sensitivity. The $S G V_{24}$ does successfully

693 capture the modularity difference between Smilodon and Canis dirus, where the

694 integration difference is large. Yet eigenvalue standard deviation continues to fail. We

695 also show that use of the correlation matrix leads to rank inflation in coordinate data; this

696 rank inflation is noise, and the correlation matrix should not be used on raw coordinate

697 data.

698 The failure of current integration metrics arises from the inclusion of a long tail of

699 non-zero eigenvalues in the eigenvalue distribution. This tail of small values will have

700 low dispersion, and eigenvalue standard deviation will be pushed lower as rank

701 deficiency increases. The geometric mean of the eigenvalue distribution (the $S G V_{24}$ ) will

702 also be pushed lower, again due to the inclusion of many small eigenvalues. These facts

703 are true on inspection but should be demonstrated; one immediate line of future research 


\section{RANK DEFICIENCY IN PHENOTYPIC COVARIANCE}

704 is a simulation study that quantifies the sensitivity of current integration metrics to

705 information rank deficiency. This paper is meant as a theoretical development and proof

706 of concept, and we do not attempt a simulation study here, although the behavior of the

707 effective and relative dispersion metrics should also be explored by simulation. The

708 seeming loss of statistical power between $D_{e}$ and $D_{r}$ deserves specific attention.

709 The use of the effective rank allows calculation of a modified form of the

710 geometric mean of the eigenvalues, which is simply the mean of the non-redundant

711 eigenvalues. We demonstrate that this new metric, $D_{e}$, successfully recovers the evolution

712 of increased modularity in Pit $61 / 67$ wolves. However, matrix rank is very sensitive to

713 sample size (Table 3), and we held sample size constant in Part 2. Therefore we introduce

714 the concept of relative dispersion, $D_{r}$, to account for differences in matrix information

715 content in Part 3. Relative dispersion accounts for dispersion in dimensionality, as well as

716 dispersion in variance, and it accounts for differences in matrix size by standardizing

717 against the permuted information content. The metric $D_{r}$ should therefore be comparable

718 among data sets, as we demonstrate via comparison with Smilodon. This comparison

719 utilizes data sets with an equal number of landmarks (14); the sensitivity of relative

720 dispersion to different landmark number requires quantification in a manner similar to the

721 analysis of classical integration metrics by Grabowski and Porto (2017).

723 Dense Semilandmarks and Latent Dispersion

724 The relative dispersion metric accounts for information rank deficiency, but it is still

725 space-specific. By this we mean that it is calculated in a shape space defined by the

726 landmarks, not the entire shape, and the relation between these two spaces is not clear. 
F. R. O'Keefe, J. A. Meachen, and P. D. Polly

727 For maximum utility, it is desirable that $D_{r}$ should be comparable among spaces defined

728 by different, arbitrary sets of landmarks. In the case of two spaces defined by the same

729 number of landmarks, as in the Canis dirus and Smilodon data compared here, one might

730 suppose that relative dispersion is comparable even if the landmarks are not homologous

731 (11 of the 14 landmarks are in fact homologous). Yet this is not true. A landmark-defined

732 space with covariance matrix $\mathbf{K}_{\mathbf{V}}$ and an effective rank based on the Shannon entropy

733 only captures the variance of the landmarks, not of the entire shape upon which those

734 landmarks occur. One way to represent the total covariance of the entire shape, $\mathbf{K}_{\mathbf{T}}$,

735 would be to sample the shape with an arbitrarily large number of landmarks, so that as

736 the magnitude of $\mathbf{V}$ increases $\mathbf{K}_{\mathbf{V}}$ would approach $\mathbf{K}_{\mathbf{T}}$. Thought of in this way, a typical

737 set of geometric morphometric data would comprise

738

$$
K_{T}=K_{V}+K_{R}
$$

Where $\mathbf{K}_{\mathbf{R}}$ is the residual covariance in the shape not captured by the landmarks in

$741 \mathbf{K}_{\mathbf{V}}$. The residual matrix $\mathbf{K}_{\mathbf{R}}$ is of unknown magnitude in all mainstream applications of

742 geometric morphometrics, and there is no guarantee that is negligible, nor that the

743 proportion $\mathbf{K}_{\mathbf{R}} / \mathbf{K}_{\mathbf{T}}$ is of constant magnitude among spaces, even if $\mathbf{K}_{\mathbf{V}}$ are of equivalent

744 effective rank. Therefore the relative dispersion metric defined above remains space-

745 specific.

746 A recent approach to estimating residual shape variance is the employment of

747 dense semilandmarks, where a curve or surface is first landmarked, and then densely

748 sampled with an increasing number of semilandmarks until the coefficient of interest 


\section{RANK DEFICIENCY IN PHENOTYPIC COVARIANCE}

749 stabilizes (Marshall et al. 2019). This procedure can be employed here. Given a shape

750 with variance $\mathbf{K}_{\mathbf{T}}$ and landmark variance $\mathbf{K}_{\mathbf{V}}$, the residual covariance $\mathbf{K}_{\mathbf{R}}$ will decrease as

$751 \quad$ V increases, so that

$$
K_{T} \approx K_{V}, \text { and } K_{R} \approx 0
$$

754 as the number of semilandmarks becomes large. The number of semilandmarks can be

755 arbitrarily large, down to the pixel or voxel resolution of the image being digitized, but in

756 practice semilandmarks need only be added until the coefficient stabilizes. Because the

757 relative dispersion metric relies on information entropy, it will scale with the information

758 added by each landmark, not by landmark number. Using this procedure allows

759 characterization of the true, or latent, dispersion of the shape;

$$
D_{l}=\lim _{v \rightarrow \infty} D_{r}
$$

763 matrix that is semilandmarked densely enough to asymptotically stabilize $D_{r}$. Classical

764 integration measures are not amenable to this procedure, because they consider all

765 eigenvalues, and as the number of landmarks increases the rank of $\mathbf{K}$ will become

766 increasingly rank deficient.

767 The latent dispersion metric should be useful for comparing shapes across

768 arbitrary landmark spaces, and for assessing the fidelity with which the original landmark

769 data capture phenotypic shape change. It is a general statement of the information content 
F. R. O'Keefe, J. A. Meachen, and P. D. Polly

770 of a shape. Calculation of this metric would allow us to state definitively that the

771 Smilodon data is tighter in shape dispersion than Canis dirus, assuming the observed

772 pattern from 14 landmarks holds up. Yet given the rank deficiency in $\mathbf{K}$ demonstrated by

773 the permuted matrices, we doubt there is sufficient residual variation not being captured

774 by the landmarks to substantially change this result. Smilodon is a highly specialized

775 hypercarnivore, and intuitively it should be more tightly adapted—and less complex-

776 than a more generalized canid like Canis dirus, whose jaw should be more information

777 rich.

778

779 Conclusions

780 -- Measures of whole-shape integration are reviewed; VanValen's realization that

781 dimensionality dispersion is a critical property is highlighted, and the Shannon, or

782 information, entropy is employed to measure it as effective rank.

783 -- Phenotypic covariance matrices for dire wolf jaws from two populations are

784 introduced: Pit 13, deposited circa 19 kya, and Pit 61/67, deposited circa 14 kya. Both

785 data matrices comprise $\mathrm{X}, \mathrm{Y}$ coordinate data for 14 identical landmarks, after Procrustes

786 superimposition.

787 -- Use of the concept of information entropy allows identification of the magnitude and

788 sources of rank deficiency in the Pit 61/67 sample. Rank deficiency is found to be high,

789 with almost half not due to phenotypic covariance.

790 -- Modularity model tests show that the only significant module is the ontogenetic one of

791 the cheek teeth relative to the jaw corpus. This module is only significant in Pit 61/67

792 wolves; its absence in Pit 13 means that Pit 13 wolves are more integrated (best fit by a 


\section{RANK DEFICIENCY IN PHENOTYPIC COVARIANCE}

793 one parameter model), while 61/67 wolves are more modular (best fit by a two parameter

794 model).

795 -- Classical metrics of whole shape integration fail to recover the evolutionary increase in

796 modularity in Pit 61/67 wolves. They fail because 1) they rely on the correlation matrix,

797 which inflates trivial variance in geometric morphometric data; and 2) they measure only

798 variance dispersion, while ignoring dimensionality dispersion.

799 -- New metrics based on the Shannon entropy-modified SGV are defined to quantify the

800 dispersion of phenotypic shapes spaces. The relative dispersion $\left(D_{r}\right)$ is a sample-size

801 corrected version of the effective dispersion $\left(D_{e}\right)$; the latent dispersion is an asymptotic

802 extension of $D_{r}$ to a dense semilandmark context. The relative dispersion is the core result

803 of this paper; we restate it from Equation 7:

804

$$
D_{r}=\frac{\sqrt[2 R e]{\prod \lambda_{R e}}}{\sqrt{\frac{1}{\pi R_{e}}}}
$$

806 -- New whole shape integration measures incorporating effective rank are successful at

807 recovering the evolution of increased modularity in 61/67 wolves, and show promise for

808 the characterization of phenotypic spaces among taxa.

\section{Supplementary Materials}

811 Data Availability Statement: The data used in this paper are available as supplementary

812 materials, and are also posted to the Dryad Digital Repository:

813 http://dx.doi.org/10.5061/dryad.d7wm37q01 
F. R. O’Keefe, J. A. Meachen, and P. D. Polly

\section{Acknowledgements}

816 We thank the curators and staff at the Tar Pit Museum, specifically John Harris, Emily

817 Lindsey, Chris Shaw, Aisling Farrell, and Gary Takeuchi, for allowing us to collect data

818 from the dire wolf specimens used in this project and for assistance of all kinds. We also

819 thank Dr. H. David Sheets for his assistance using the IMP programs, including

820 pcagen_7a. We thank the Graduate College for the Marshall University Summer Thesis

821 Award and the Marshall University Drinko Research Fellowship to FRO for funding

822 towards this project. Special thanks are due to Emily Lindsey, John Southon, Wendy

823 Binder, and Larisa DeSantis for input on early versions of this manuscript, and to Bryan

824 Carstens, Lauren Esposito, and two anonymous reviewers for thorough and very helpful

825 input during the review process. Work by FRO was funded in part by NSF EAR-SGP

8261757236 (Project SABER), and by a Drinko Distinguished Research Fellowship to FRO.

827 We grudgingly thank Thom Yorke.

828 


\section{RANK DEFICIENCY IN PHENOTYPIC COVARIANCE}

\section{$829 \quad$ Literature Cited}

830 Adams, D. C. 2016. Evaluating modularity in morphometric data: challenges with the RV 831 coefficient and a new test measure. Methods in Ecology and Evolution 7(5): 565832572.

833 Adams, D., Collyer, M., and Kaliontzopoulou, A. 2020. Geomorph: Software for 834 geometric morphometric analyses. R package version 3.2.1. https://cran.r835 project.org/package=geomorph.

836 Binder, W. J., Thompson, E. N., \& Van Valkenburgh, B. 2002. Temporal variation in

837 tooth fracture among Rancho La Brea dire wolves. Journal of Vertebrate $838 \quad$ Paleontology 22(2): 423-428.

839 Binder, W.J. and Van Valkenburgh, B. 2010. A comparison of tooth wear and breakage

840 in Rancho La Brea sabertooth cats and dire wolves across time. Journal of $841 \quad$ Vertebrate Paleontology 30: 255-261.

842 Bookstein, F. L. 1997. Morphometric tools for landmark data: geometry and biology. $843 \quad$ Cambridge University Press.

844 Brannick, A.L., Meachen, J.A., and O’Keefe, F.R. 2015. Microevolution of jaw shape in

845 the dire wolf, Canis dirus, at Rancho La Brea. La Brea and beyond: The

846 paleontology of asphalt-preserved biotas, ed. J. M. Harris. Natural History

847 Museum of Los Angeles County, Science Series 42: 23-32.

848 Bunea, F., She, Y., and Wegkamp, M. H. 2011. Optimal selection of reduced rank

849 estimators of high-dimensional matrices. The Annals of Statistics 39(2): 1282-

$850 \quad 1309$. 
F. R. O’Keefe, J. A. Meachen, and P. D. Polly

851 Cangelosi, R. and Goriely, A. 2007. Component retention in principal component

852 analysis with application to cDNA microarray data. Biology Direct 2(1): 1-21.

853 Cheverud, J. M. 1982. Phenotypic, genetic, and environmental morphological integration

854 in the cranium. Evolution 36: 499-516.

855 Cheverud, J. M. 1996. Developmental integration and the evolution of pleiotropy.

$856 \quad$ American Zoologist 36(1): 44-50.

857 Curth, S., Fischer, M. S., and Kupczik, K. 2017. Patterns of integration in the canine skull: an inside view into the relationship of the skull modules of domestic dogs and wolves. Zoology 125: 1-9.

862 Grabowski, M. and Porto, A. 2017. How many more? Sample size determination in studies of morphological integration and evolvability. Methods in Ecology and

865 Goswami, A., Smaers, J. B., Soligo, C. and Polly, P. D. 2014. The macroevolutionary

866 consequences of phenotypic integration: from development to deep time. Philosophical Transactions of the Royal Society B: Biological Sciences, 369(1649): 20130254.

869 Goswami, A., Binder, W. J., Meachen, J., \& O’Keefe, F. R. 2015. The fossil record of 870 phenotypic integration and modularity: A deep-time perspective on 871 developmental and evolutionary dynamics. Proceedings of the National Academy $872 \quad$ of Sciences 112(16): 4891-4896. 


\section{RANK DEFICIENCY IN PHENOTYPIC COVARIANCE}

873 Goswami, A. and Polly, P.D. 2010. Methods for studying morphological integration and

874 modularity. The Paleontological Society Papers 16: 213-243.

875 Hine, E., and Blows, M. W. 2006. Determining the effective dimensionality of the

876 genetic variance-covariance matrix. Genetics 173(2): 1135-1144.

877 Horvath, S. 2011. Weighted network analysis: applications in genomics and systems

$878 \quad$ biology. Springer Science \& Business Media.

879 Jolicoeur, P. 1963. Note: the multivariate generalization of the allometry equation.

$880 \quad$ Biometrics 19(3): 497-499.

881 Klingenberg, C. P. 2008. Morphological integration and developmental modularity.

882 Annual Review of Ecology, Evolution and Systematics 39: 115-132.

883 Klingenberg CP. 2010. Evolution and development of shape: integrating quantitative

884 approaches. Nature Reviews Genetics 11(9): 623-35.

885 Klingenberg CP. 2013. Cranial integration and modularity: insights into evolution and

886 development from morphometric data. Hystrix, the Italian Journal of Mammalogy

$887 \quad 24(1): 43-58$.

888 Marshall, A. F., Bardua, C., Gower, D. J., Wilkinson, M., Sherratt, E., \& Goswami, A.

889 2019. High-density three-dimensional morphometric analyses support conserved

$890 \quad$ static (intraspecific) modularity in caecilian (Amphibia: Gymnophiona)

891 crania. Biological Journal of the Linnean Society 126(4): 721-742.

892 Meachen, J.A., O'Keefe, F.R., and Sadleir, R.W. 2014. Evolution in the sabre-tooth cat,

893 Smilodon fatalis, in response to Pleistocene climate change. Journal of

894 Evolutionary Biology 27: 714-723. 
F. R. O’Keefe, J. A. Meachen, and P. D. Polly

895 Meloro, C. 2012. Mandibular shape correlates of tooth fracture in extant Carnivora:

896 implications to inferring feeding behavior of Pleistocene predators. Biological

897 Journal of the Linnean Society 106: 70-80.

898 Najarzadeh, D. 2019. Testing equality of standardized generalized variances of k

899 multivariate normal populations with arbitrary dimensions. Statistical Methods \&

$900 \quad$ Applications 28(4): 593-623.

901 O'Keefe, F. R., Rieppel, O., \& Sander, P. M. 1999. Shape disassociation and inferred

902 heterochrony in a clade of pachypleurosaurs (Reptilia, Sauropterygia).

$903 \quad$ Paleobiology 25: 504-517.

904 O'Keefe, F. R., Binder, W. J., Frost, S. R., Sadlier, R. W. and Van Valkenburgh, B. 2014.

905 Cranial morphometrics of the dire wolf, Canis dirus, at Rancho La Brea: temporal

906 variability and its links to nutrient stress and climate. Palaeontologia Electronica.

907 O'Keefe F. R., Meachen J, Fet E. V., Brannick A. 2016. Ecological determinants of clinal

908 morphological variation in the cranium of the North American gray wolf. Journal

909 of Mammalogy 94(6):1223-36.

910 Olson, E.C. and Miller, R.L., 1958. Morphological Integration. University of Chicago

$911 \quad$ Press (reprint, 1999).

912 Pavlicev, M., Cheverud, J. M., \& Wagner, G. P. 2009. Measuring morphological

913 integration using eigenvalue variance. Evolutionary Biology 36(1): 157-170.

914 R Core Team, 2014. R: A language and environment for statistical computing.

915 Rohlf, F. 2013. tpsDig2, Stony Brook: State University of New York.

916 Roy, O. and Vetterli, M. 2007. The effective rank: A measure of effective dimensionality.

917 In 2007 15th European Signal Processing Conference: 606-610. IEEE. 


\section{RANK DEFICIENCY IN PHENOTYPIC COVARIANCE}

918 Segura, V., Cassini, G. H., Prevosti, F. J., \& Machado, F. A. 2020. Integration or

919 Modularity in the Mandible of Canids (Carnivora: Canidae): a Geometric

920 Morphometric Approach. Journal of Mammalian Evolution

$921 \quad$ doi.org/10.1007/s10914-020-09502-z.

922 SenGupta, A., 1987. Tests for standardized generalized variances of multivariate normal

923 populations of possibly different dimensions. Journal of Multivariate Analysis

$924 \quad 23(2): 209-219$.

925 Shannon, C.E. 1948. A mathematical theory of communication. The Bell System

$926 \quad$ Technical Journal 27(3): 379-423.

927 Stock, C. and Harris, J.M. 1992. Rancho La Brea: A Record of Pleistocene Life in

928 California. Science Series No. 37. Natural History Museum of Los Angeles

929 County, Los Angeles, CA.

930 Van Valen, L. 1974. Multivariate structural statistics in natural history. Journal of

931 Theoretical Biology 45(1): 235-247.

932 Van Valkenburgh, B. 1988. Incidence of tooth fracture among large, predatory mammals.

$933 \quad$ American Naturalist 13: 291-302.

934 Van Valkenburgh, B. 2009. Costs of carnivory: tooth frac- ture is Pleistocene and recent

935 carnivores. Biological Journal of the Linnean Society 96: 68-81.

936 Wagner, G. P. 1984. On the eigenvalue distribution of genetic and phenotypic dispersion

937 matrices: evidence for a nonrandom organization of quantitative character

938 variation. Journal of Mathematical Biology 21: 77-95.

939 Wilks, S. S. 1932. Certain generalizations in the analysis of variance. Biometrika: 471-

940494. 

available under aCC-BY-NC-ND 4.0 International license.

F. R. O’Keefe, J. A. Meachen, and P. D. Polly

941 Zelditch, M.L., Swiderski, D.L. and Sheets, H.D. (2012). Geometric morphometrics for

942 biologists: a primer. Academic Press.

943

944 


\section{RANK DEFICIENCY IN PHENOTYPIC COVARIANCE}

\section{$945 \quad$ Appendix 1}

946

947

948

$R$ Code for Effective Dispersion Metrics, 1. Code for calculating permuted SVG, Effective

949

950

951

952

953

954

955

956

957

958

959

960

961

962

963

Rank, and Effective Dispersion

964

\#attach necessary libraries

library(dplyr)

library(broom)

library(readxl)

\#Define a variable containing the GPA X,Y landmark data. The data is read in as a data frame from an Excel file.

GPALMData <- read_excel(" /Desktop/C dirus 14 LM/CDirus6167LM.xlsx")

View(GPALMData)

965

EffRank=matrix $($ nrow $=1$, ncol $=0)$

$\mathrm{SGV}=\operatorname{matrix}($ nrow $=1, \mathrm{ncol}=0)$

966

EffRankSGV=matrix $($ nrow $=1$, ncol $=0)$

967

968

EigenSumRec $=$ matrix $($ nrow $=1, \mathrm{ncol}=0)$

969

\#Start permutation loop

970

971

972

973

974

975

976

977

for (i in 1:1000)\{

978

979

980

\#Permute rows in each variable column vector independently for permutation test.

LMPrune $=($ GPALMData [3:30] $)$

PermLM = apply(LMPrune, 2, sample, 83, replace=FALSE)

982

983

984

985

986

\#Define variable that is the covariance matrix of the above. Be sure to specify only the landmark columns in the data frame.

$\mathrm{CVLM}=\operatorname{cov}($ PermLM $)$

\#toggle for cov/cor

\#Define object that is the eigenanalysis of the covariance matrix.

CVLMeigen=eigen $(\mathrm{CVLM})$

EigenVal<-CVLMeigen\$values

\#Define the summation of eigenvalues from the eigenanalysis.

EigenSum=sum(EigenVal)

\#Create vector of scaled eigenvalues

990 PofK<-EigenVal/EigenSum 
F. R. O'Keefe, J. A. Meachen, and P. D. Polly

991

992

993

994

995

996

997

998

999

1000

1001

1002

1003

1004

1005

1006

1007

1008

1009

1010

1011

1012

1013

1014

1015

1016

1017

1018

1019

1020

1021

1022

1023

1024

1025

1026

1027

1028

1029

1030

1031

1032

1033

1034

1035

1036
\#Creat vector of $\operatorname{lnPofK}$

$\ln$ PofK $<-\log ($ PofK $)$

Product $<-\operatorname{lnPofK} *$ PofK

SumProduct<-sum(Product, na.rm=TRUE)

ShannonEntropy <- - $1 *$ SumProduct

EffectiveRank<-exp(ShannonEntropy)

\#Begin SGV24 calculation

EigenProduct<-prod(EigenVal[1:24])

StandGenVar<-(EigenProduct $)^{\wedge}(1 / 24)$

SGV<-cbind(SGV, StandGenVar)

\#Begin ReSGV calculation

ERInteger<-as.integer(EffectiveRank)

EigenTrim<-Eigen Val[1:ERInteger]

FractRankVar<-EigenVal[ERInteger+1]*(EffectiveRank-ERInteger)

ProductEffRankEigen<-prod(EigenTrim)*FractRankVar

EffectiveRankSGV<-(ProductEffRankEigen $)^{\wedge}(1 /$ EffectiveRank $)$

EffRank <-cbind(EffRank, EffectiveRank)

EffRankSGV <-cbind(EffRankSGV, EffectiveRankSGV)

EigenSumRec <-cbind(EigenSumRec, EigenSum)

cat("\014")

print(i)

\}

\#calculate and print st deviation and mean for each parameter

$\operatorname{print}(m e a n($ EigenSumRec $)$ )

print (sd(EigenSumRec)) 
bioRxiv preprint doi: https://doi.org/10.1101/2020.07.23.218289; this version posted March 15, 2021. The copyright holder for this preprint (which was not certified by peer review) is the author/funder, who has granted bioRxiv a license to display the preprint in perpetuity. It is made available under aCC-BY-NC-ND 4.0 International license.

RANK DEFICIENCY IN PHENOTYPIC COVARIANCE

1037

$1038 \operatorname{print}($ mean(EffRank))

1039 print (sd(EffRank))

1040

1041 print (mean(SGV))

1042 print $(\mathrm{sd}(\mathrm{SGV}))$

1043

1044 print (mean(EffRankSGV))

1045 print (sd(EffRankSGV))

1046 
F. R. O'Keefe, J. A. Meachen, and P. D. Polly

1047

1048

1049

1050

1051

1052

1053

1054

1055

1056

1057

1058

1059

1060

1061

1062

1063

1064

1065

1066

1067

1068

1069

1070

1071

1072

1073

1074

1075

1076

1077

1078

1079

1080

1081

1082

1083

1084

1085

1086

1087

1088

1089

1090

1091

1092

$R$ Code for Effective Dispersion Metrics, 2. Code for calculating SVG, Effective Rank, and Effective Dispersion

\#attach necessary libraries

library(dplyr)

library(broom)

library(readxl)

\#Define a variable containing the $\mathrm{X}, \mathrm{Y}$ landmark data. The data is read in as a data frame from an Excel file.

GPALM <- read_excel(" /Desktop/C Dirus 14 LM/CDirus13LM.xlsx")

View(GPALM)

\#initialize variables

EffRank=matrix $($ nrow $=1$, ncol $=0)$

$\mathrm{SGV}=$ matrix $($ nrow $=1, \mathrm{ncol}=0$ )

EffRankSGV=matrix (nrow $=1$, ncol $=0$ )

EigenSumRec $=$ matrix $($ nrow $=1$, ncol $=0)$

EffDispersion $=$ matrix $($ nrow $=1$, ncol $=0)$

\#Start bootstrap loop

for (i in 1:10000)\{

bootsample=sample_n $($ GPALM, 36, replace $=$ FALSE $)$

\#edit for $\mathrm{n}$ and $\mathrm{T} / \mathrm{F}$

\#Define variable that is the covariance matrix of the above. Be sure to specify only the landmark columns in the data frame.

CVLM= $\operatorname{cov}($ bootsample[3:30])

\#toggle cov/cor

\#Define object that is the eigenanalysis of the covariance matrix.

CVLMeigen=eigen $($ CVLM $)$

EigenVal<-CVLMeigen\$values

\#Define the summation of eigenvalues from the eigenanalysis.

EigenSum=sum(EigenVal)

\#Create vector of scaled eigenvalues

PofK $<$-EigenVal/EigenSum

\#Creat vector of $\operatorname{lnPofK}$ and calculate effective rank

$\ln$ PofK $<-\log ($ PofK $)$ 


\section{RANK DEFICIENCY IN PHENOTYPIC COVARIANCE}

1093

1094

1095

1096

1097

1098

1099

1100

1101

1102

1103

1104

1105

1106

1107

1108

1109

1110

1111

1112

1113

1114

1115

1116

1117

1118

1119

1120

1121

1122

1123

1124

1125

1126

1127

1128

1129

1130

1131

1132

1133

1134

1135

1136

1137

1138
Product $<-\ln$ PofK*PofK

SumProduct $<$-sum(Product, na.rm=TRUE)

ShannonEntropy $<--1 *$ SumProduct

EffectiveRank<-exp(ShannonEntropy)

\#Begin SGV24 calculation

EigenProduct $<-$ prod(EigenVal[1:24])

StandGenVar<-(EigenProduct $)^{\wedge}(1 / 24)$

SGV<-cbind(SGV, StandGenVar)

\#Begin ReSGV calculation

ERInteger $<$-as.integer(EffectiveRank)

EigenTrim<-EigenVal[1:ERInteger]

FractRankVar<-EigenVal[ERInteger+1]*(EffectiveRank-ERInteger)

ProductEffRankEigen<-prod(EigenTrim)*FractRankVar

EffectiveRankSGV<-(ProductEffRankEigen $)^{\wedge}(1 /$ EffectiveRank $)$

\#Make Effective Dispersion

EffDisperse $<$-sqrt(EffectiveRankSGV)

EffRank <-cbind(EffRank, EffectiveRank)

EffRankSGV <-cbind(EffRankSGV, EffectiveRankSGV)

EigenSumRec <-cbind(EigenSumRec, EigenSum)

EffDispersion <-cbind(EffDispersion, EffDisperse)

cat("1014")

$\operatorname{print}(\mathrm{i})$

\}

\#calculate and print st deviation and mean for each parameter 
F. R. O'Keefe, J. A. Meachen, and P. D. Polly

1139

$1140 \operatorname{print}(m e a n($ EigenSumRec $))$

1141 print (sd(EigenSumRec))

1142

1143

1144

1145

1146

1147

1148

1149

1150

1151

1152

1153

$\operatorname{print}(m e a n(E f f R a n k))$

print (sd(EffRank))

1154

1155

print (mean(SGV))

print $(\mathrm{sd}(\mathrm{SGV}))$

print (mean(EffRankSGV))

print (sd(EffRankSGV))

print (mean(EffDispersion))

print (sd(EffDispersion)) 


\section{RANK DEFICIENCY IN PHENOTYPIC COVARIANCE}

$1156 R$ Code for Effective Dispersion Metrics, 3. Code for calculating eigenvalue standard

1157 deviation

1158

1159

1160

1161

\#attach required packages

library(dplyr)

library(broom)

1163

1164

1165

1166

\#Define a variable containing the $\mathrm{X}, \mathrm{Y}$ landmark data. The data is read in as a data frame from an Excel file.

\section{GPALM <- read_excel(" /Desktop/C dirus 14 LM/CDirus6167LM.xlsx")}

View(CDirus6167AllomResidLM)

BSSDEigen $=$ matrix $($ nrow $=1, \mathrm{ncol}=0)$

\#Start bootstrap loop

for (i in 1:1000)\{ bootsample=sample_n $($ GPALM, 83, replace $=$ FALSE $)$ \#edit $\mathrm{n}$, bootstrap or not

\#Define variable that is the covariance matrix of the above. Be sure to specify only the landmark columns in the data frame.

CVLM= cor(bootsample[3:30]) \#toggle cov/cor

\#Define object that is the eigenanalysis of the covariance matrix.

CVLMeigen=eigen $($ CVLM $)$

EigenVal<-CVLMeigen\$values

EigenValTrim<-EigenVal[1:24]

EigenMean <-(sum(EigenValTrim) $) / 24$

EigenValTrimMean<-EigenValTrim/EigenMean

EigenK $<-(\text { abs }(\text { EigenValTrimMean-1) }))^{\wedge}$

Eigenvar <- $(\operatorname{sum}($ EigenK $)) / 24$

SDEigen <- sqrt(Eigenvar) $/ 4.7958$

BSSDEigen <-cbind(BSSDEigen, SDEigen)

1201 
bioRxiv preprint doi: https://doi.org/10.1101/2020.07.23.218289; this version posted March 15, 2021. The copyright holder for this preprint (which was not certified by peer review) is the author/funder, who has granted bioRxiv a license to display the preprint in perpetuity. It is made available under aCC-BY-NC-ND 4.0 International license.

F. R. O’Keefe, J. A. Meachen, and P. D. Polly

1202

1203 \#calculate and pring st deviation and mean for each parameter

1204

1205

$\operatorname{print}($ mean(BSSDEigen))

1206

1207

print (sd(BSSDEigen))

1208 


\section{RANK DEFICIENCY IN PHENOTYPIC COVARIANCE}

\section{Appendix 2}

1210 Testing the dire wolf data sets for modularity requires specifying candidate models. We

1211 use a heuristic approach based on the multivariate allometry vectors of interlandmark

1212 distances. Initial analysis consisted of calculating the interlandmark distances from the

1213 raw digitized landmark data LM (Figure A1). These distances were then evaluated for

1214 multivariate allometry by calculating the first principal component vector of the

1215 covariance matrix of $\ln$-transformed distances; this procedure yields the multivariate

1216 allometry vector (Jolicoeur, 1963; O'Keefe et al., 1999; O'Keefe et al., 2013). Confidence

1217 intervals for the allometry vector coefficients where calculated by bootstrapping the input

1218 data (10000 replicates) in the $\mathrm{R}$ core package ( $\mathrm{R}$ Core Team, 2014). The eigenvalues of

1219 the full 13-measure multivariate allometry vector were unusual, necessitating additional

1220 runs with some variables removed; all runs are reported in Table A1.

1221 The multivariate allometry vector for all 13 interlandmark distances is odd, with a

1222 single variable scoring very highly (the distance between the canine and the first

1223 premolar, landmarks 3-4), while the rest show significantly negative coefficients. This

1224 pattern indicates that the c1-p1 distance is highly variable, and that this variance is not

1225 correlated with any other variable. Further analysis shows that the c1-p1 distance crosses

1226 the boundary between two modules, accounting for its unique loading. We therefore

1227 discarded that distance and ran the analysis again with 12 distances. The resulting vectors

1228 for both populations are strongly positively allometric for two variables, the

1229 anteroposterior width of the canine (1-2) and the length of the condyloid process (9-10).

1230 Lengths associated with the other teeth are negatively allometric (Table A1). The

1231 covariation of canine width and condyloid process length suggests a functional unit, and 
F. R. O'Keefe, J. A. Meachen, and P. D. Polly

1232 provides one hypothesis for a testable module in the modularity tests. The lengths of the

1233 other teeth are all negatively allometric, as are the landmarks on the inferior margin on

1234 the jaw that are linked to tooth location. This finding of negative dental allometry in the

1235 cheek teeth has been documented before, both in Canis lupus (O'Keefe et al., 2016) and

1236 in Canis dirus (O'Keefe et al., 2014). Negative dental allometry is a consequence of the

1237 adult teeth forming early in ontogeny, erupting before the end of the first year, while full

1238 somatic growth is not attained until the end of the second year (O'Keefe et al., 2016).

1239 This negative dental allometry in the cheek teeth suggests a second module, comprising

1240 the lengths of the premolar arcade, the carnassial, and $\mathrm{m} 2$. We therefore specify three

1241 candidate modules for testing. They are the condyloid process and canine, the cheek

1242 teeth, and the rest of the jaw. The modularity models tested in Table 1 are permutations

1243 of these modules. We also note a significant allometric difference in the coronoid process

1244 in Pit 13 wolves; this is consistent with sexual diomorphism (O'Keefe et at., 2016), and

1245 we also tested modules including landmark 8 to test for this.

1246 A valid criticism of our heuristic approach is that it does not test the model space

1247 exhaustively. The space of possible modules in a shape of 14 landmarks is $2^{14}-1$, or

1248 16,383, small enough to allow exhaustive optimization using maximum likelihood. An

1249 alternative approach is weighted network analysis (Horvath, 2011), an effective method

1250 of model induction used with genomic data. We believe that the parts of the model space

1251 tested here capture all plausible candidate models, based on our analysis of the

1252 multivariate allometry vectors, and on subsidiary principal components analysis of the

1253 landmark data.

1254 


\section{RANK DEFICIENCY IN PHENOTYPIC COVARIANCE}

\begin{tabular}{cccccc}
\hline Distance & Pit 13 & Pit 61/67 & $+/-, n=36 \mathbf{j}$ & All & $+/-$, BS \\
\hline $1-2$ & 0.6543 & 0.3366 & $0.105^{* *}$ & 0.4889 & $0.056^{* *}$ \\
\hline $3-4$ & 0.0625 & 0.1503 & $0.036^{* *}$ & 0.1883 & $0.017^{* *}$ \\
\hline $4-6$ & 0.1339 & 0.1721 & 0.037 & 0.1500 & $0.022^{* *}$ \\
\hline $6-7$ & 0.1291 & 0.1561 & 0.071 & 0.1439 & $0.060^{* *}$ \\
\hline $7-8$ & 0.0706 & 0.2486 & $0.034^{* *}$ & 0.1927 & $0.020^{* *}$ \\
\hline $8-9$ & 0.0982 & 0.2457 & $0.038^{* *}$ & 0.2043 & $0.018^{* *}$ \\
\hline $9-10$ & 0.4183 & 0.4479 & 0.096 & 0.5682 & $0.043^{* *}$ \\
\hline $10-11$ & 0.2218 & 0.3494 & $0.056^{* *}$ & 0.2711 & 0.033 \\
\hline $11-12$ & 0.1140 & 0.2324 & $0.048^{* *}$ & 0.2105 & $0.023^{* *}$ \\
\hline $12-13$ & 0.1408 & 0.1562 & 0.041 & 0.1531 & $0.029^{* *}$ \\
\hline $13-14$ & 0.0593 & 0.1539 & $0.041^{* *}$ & 0.1842 & $0.012^{* *}$ \\
\hline $14-1$ & 0.5082 & 0.4540 & 0.133 & 0.2967 & 0.066 \\
\hline Isometry & 0.289 & 0.289 & & 0.289 & \\
\hline
\end{tabular}

Table 1. Allometry vectors calculated from interlandmark distances on Canis

1256 dirus dentaries as shown in Figure 1. Three vectors are shown; that for Pit 13, for Pit

$125761 / 67$, and for the pooled sample. We also jackknifed the Pit 61/67 sample to establish a

1258 confidence interval; stars in this column indicate significant differences from Pit 13. The

1259 pooled vector was also bootstrapped, and stars in this column indicate differences from

1260 isometry in that vector. The isometric coefficient value for each vector is shown at

1261 bottom; confidence intervals for coefficient values are one standard deviation of 1000 


\section{F. R. O'Keefe, J. A. Meachen, and P. D. Polly}

1262 bootstrap replicates of each vector. Stars are 2 sigma. The heat maps in Figure 1A are

1263 indexed to the pooled coefficients.

1264

A

$-0.563$
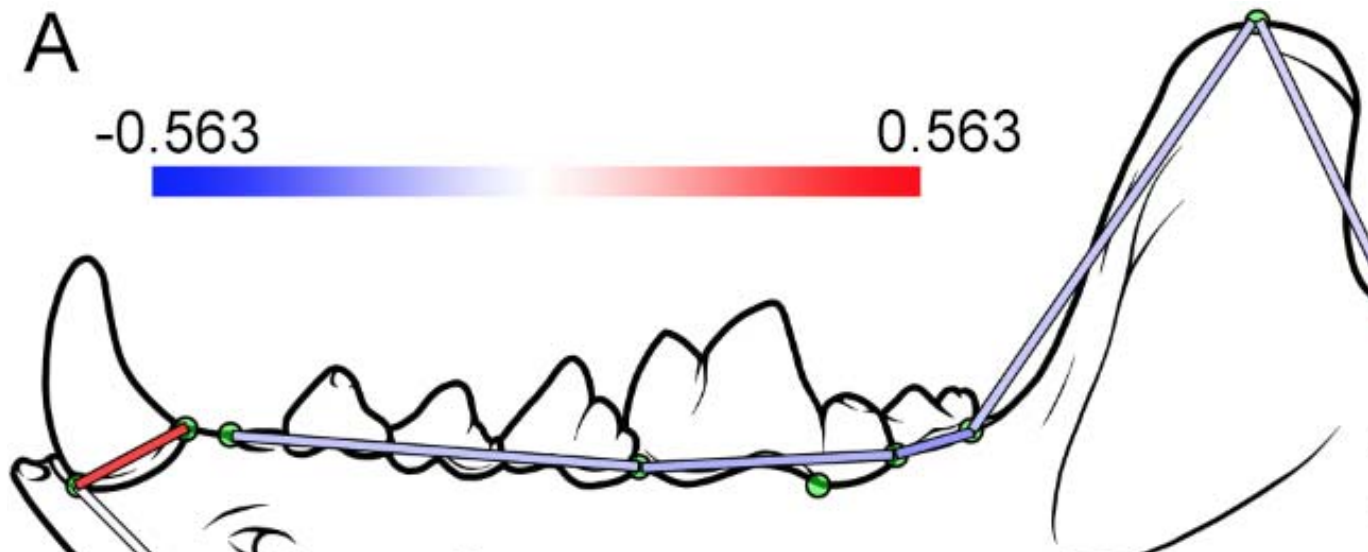

available under aCC-BY-NC-ND 4.0 International license.

\section{RANK DEFICIENCY IN PHENOTYPIC COVARIANCE}

1270 measure allometry vector. These different allometries suggest three different modules:

1271 the condoloid process and the canine, the check teeth, and the rest of the jaw. 